\title{
Supersymmetric field theories and geometric Langlands: The other side of the coin
}

\author{
Aswin Balasubramanian, Jörg Teschner \\ Department Mathematik, \\ Universität Hamburg, \\ Bundesstrae 55, \\ 20146 Hamburg, Germany \\ and: \\ DESY theory, \\ Notkestrasse 85, \\ 22607 Hamburg, Germany
}

\begin{abstract}
This note announces results on the relations between the approach of Beilinson and Drinfeld to the geometric Langlands correspondence based on conformal field theory, the approach of Kapustin and Witten based on $N=4 \mathrm{SYM}$, and the AGT-correspondence. The geometric Langlands correspondence is described as the Nekrasov-Shatashvili limit of a generalisation of the AGT-correspondence in the presence of surface operators. Following the approaches of Kapustin - Witten and Nekrasov - Witten we interpret some aspects of the resulting picture using an effective description in terms of two-dimensional sigma models having Hitchin's moduli spaces as target-manifolds.
\end{abstract}

\section{Introduction}

Some remarkable connections between supersymmetric gauge theories and conformal field theory (CFT) have been discovered in the last few years. One of the most explicit connections was discovered by Alday, Gaiotto and Tachikawa [1], nowadays often referred to as AGTcorrespondence. It overlaps with another family of results for which Nekrasov and collaborators have introduced the name BPS/CFT-correspondence, see [2] for the first in a series of 
papers on this subject and references to previous work in this direction. An older development, the relations between the $N=4$ super-Yang-Mills theory (SYM) and the geometric Langlands correspondence exhibited by Kapustin and Witten [3], naturally fits into the emerging picture [4. 5]. One may note, however, that the approach of Beilinson and Drinfeld to the geometric Langlands correspondence has close connections to CFT, see [6] for a review and further references, which are not obvious in the approach of Kapustin and Witten. Some connections between these subjects have been discussed in [4, 7, 5, 8], and the recent work [9, 10] indicates that at least a part of these relations admit a further deformation, motivated by supersymmetric gauge and string theory. However, the picture still seems to be incomplete in many respects.

This note, prepared for the proceedings of String-Math 2016, announces results shedding some light on the relations between the approaches to the geometric Langlands correspondence of Beilinson and Drinfeld, Kapustin and Witten, and the AGT-correspondence. We will mostly restrict attention to the cases where the underlying Lie-algebra is $\mathfrak{s l}_{2}$ in order to keep the length of this note within reasonable bounds. The results described here are part of a larger project being pursued by the authors together with Ioana Coman-Lohi. A series of publications containing more details, an extended discussion of mathematical aspects, and a discussion of the higher rank cases is in preparation.

In the main text we will freely use several standard definitions and results concerning Hitchin's moduli spaces. A very brief summary is collected in Appendix A in the form of a glossary. If a glossary entry exists for a term, its first occurrence will appear with a superscript as in term ${ }^{g)}$.

\section{Review}

\subsection{What is the geometric Langlands correspondence?}

The geometric Langlands correspondence is often loosely formulated as a correspondence which assigns $\mathcal{D}$-modules on $\operatorname{Bun}_{G}$ to ${ }^{L} G$-local systems ${ }^{g)}$ on a Riemann surface $C$. ${ }^{L} G$ is the Langlands dual group of a simple complex Lie group $G$. The ${ }^{L} G$-local systems appearing in this correspondence can be represented by pairs $\left(\mathcal{E}, \nabla^{\prime}\right)$ composed of a holomorphic ${ }^{L} G$-bundle $\mathcal{E}$ with a holomorphic connection $\nabla^{\prime}$, or equivalently by the representations $\rho$ of the fundamental group $\pi_{1}(C)$ defined from the holonomies of $\left(\mathcal{E}, \nabla^{\prime}\right)$. We will mostly be interested in the case of irreducible $^{L} G$-local systems. The corresponding $\mathcal{D}$-modules on $\operatorname{Bun}_{G}$ can be described more concretely as systems of partial differential equations taking the form of eigenvalue equations $D_{i} f=E_{i} f$ for a family of differential operators $D_{i}$ on $\operatorname{Bun}_{G}$ obtained by quantising the Hamiltonians of Hitchin's integrable system. A more ambitious version of the geometric Langslands correspondence has been formulated in [11] in which it becomes necessary to extend it to 
certain classes of reducible local systems.

Some of the original approaches to the geometric Langlands correspondence start from the

cases where the ${ }^{L} G$-local systems are opers ${ }^{g}$, pairs $\left(\mathcal{E}, \nabla^{\prime}\right)$ in which $\nabla^{\prime}$ is gauge-equivalent to a certain standard form. The space of opers forms a Lagrangian subspace in the moduli space of all local systems. The CFT-based approach of Beilinson and Drinfeld constructs for each oper an object in the category of $\mathcal{D}$-modules on $\mathrm{Bun}_{G}$ as conformal blocks of the affine Lie algebra $\hat{\mathfrak{g}}_{k}$ at the critical level $k=-h^{\vee}$. The Ward-identities characterising the conformal blocks equip the sheaves of conformal blocks with a $\mathcal{D}$-module structure. The universal enveloping algebra $\mathcal{U}\left(\hat{\mathfrak{g}}_{k}\right)$ has a large center at $k=-h^{\vee}$, isomorphic to the space of ${ }^{L} \mathfrak{g}$-opers on the formal disc [12]. This can be used to show that the $\mathcal{D}$-module structure coming from the Ward identities can be described by the system of eigenvalue equations $D_{i} f=E_{i} f$ for the quantised Hitchin Hamiltonians, with eigenvalues $E_{i}$ parameterising the choice of opers [6].

There exists an extension of the Beilinson-Drinfeld construction of the geometric Langlands correspondence described in [6, Section 9.6] from the case of opers to general irreducible local systems. It is based on the fact that such local systems are always gauge-equivalent to opers with certain extra singularities [13]. The construction of Beilinson and Drinfeld associates to such opers conformal blocks of $\hat{\mathfrak{g}}_{-h^{\vee}}$ with certain degenerate representations induced from the finitedimensional representations of $\mathfrak{g}$ inserted at the extra singularities. We may in this sense regard the geometric Langlands correspondence for general irreducible local systems as an extension of the correspondence that exists for ordinary, non-singular opers. This point of view will turn out to be natural from the perspective we will propose in this paper. Let us also remark that the construction of Beilinson-Drinfeld plays an important role in the outline given in [14] for a proof of the strengthened geometric Langlands conjecture formulated in [11].

For future reference let us note that the Beilinson-Drinfeld construction describes the $\mathcal{D}$ modules appearing in the geometric Langlands correspondence as spaces of conformal blocks which, naturally being fibered over $\mathrm{Bun}_{G}$ on the one hand, are furthermore getting fibered over the spaces of irreducible ${ }^{L} G$-local systems on the other hand.

\subsection{Geometric Langlands correspondence - approach of Kapustin-Witten}

The variant of the geometric Langlands correspondence proposed by Kapustin and Witten [3] is based on the consideration of $N=4 \mathrm{SYM}$ theory with gauge group $G_{c}$, a compact real form of $G$, on four-manifolds of the form $\Sigma \times C$, where $C$ is a Riemann surface. Compactification on $C$ allows one to represent the topologically twisted version of $N=4$ SYM effectively by a topologically twisted $2 d$ sigma-model with target being the Hitchin moduli space ${ }^{(g)} M_{H}(G)$ on $\Sigma$. The complete integrability of the Hitchin moduli space, as is manifest in the description of 
$M_{H}(G)$ as a torus fibration, allows one to describe the consequences of the S-duality of $N=4$ SYM theory as the SYZ mirror symmetry relating the $2 d$ sigma-models with target $M_{H}(G)$ and $M_{H}\left({ }^{L} G\right)$, respectively.

In order to relate this to the geometric Langlands correspondence, Kapustin and Witten consider the cases when $\Sigma=R \times I, I=[0, \pi]$. Supersymmetric boundary condition of $N=4 \mathrm{SYM}$ theory will upon compactification on $C$ define boundary conditions of the $\mathcal{N}=(4,4)$ sigma model with target $\mathcal{M}_{H}(G)$ on $\Sigma$. Let $\mathfrak{B}$ be the category having as objects boundary conditions $B$ called branes preserving the maximal number of supersymmetries, with morphisms being the spaces of "open string" states $\operatorname{Hom}_{\mathcal{M}_{H}(G)}\left(\mathrm{B}_{1}, \mathrm{~B}_{2}\right)$ of the sigma model on the strip $\mathbb{R} \times I$, having associated boundary conditions $B_{1}$ and $B_{2}$ to the boundaries $\mathbb{R} \times\{0\}$ and $\mathbb{R} \times\{\pi\}$, respectively.

A distinguished role is played by the so-called canonical coisotropic brane $B_{c c}$ [3, 4]. The vector space $\mathcal{A}_{\mathrm{cc}}=\operatorname{Hom}_{\mathcal{M}_{H}(G)}\left(\mathrm{B}_{\mathrm{cc}}, \mathrm{B}_{\mathrm{cc}}\right)$ has a natural algebra structure with product defined by "joining open strings". The spaces $\mathcal{H}(\mathrm{B})=\operatorname{Hom}_{\mathcal{M}_{H}(G)}\left(\mathrm{B}_{\mathrm{cc}}, \mathrm{B}\right)$ are left modules over the algebra $\mathcal{A}_{\mathrm{cc}}$ with action defined by "joining open strings" from $\mathcal{A}_{\mathrm{cc}}$ on the left boundary of the strip $I$. Kapustin and Witten argue that the algebra $\mathcal{A}_{\text {cc }}$ contains the algebra of global differential operators on $\operatorname{Bun}_{G}$. It follows that the spaces $\mathcal{H}(\mathrm{B})$ represent $\mathcal{D}$-modules on $\operatorname{Bun}_{G}$.

The reduction of Wilson- and 't Hooft line operators with support on $\mathbb{R} \times\{x\} \times P, x \in I, P \in$ $C$, to the two dimensional TQFT defines natural functors on the category of branes, inducing modifications of the spaces $\mathcal{H}(\mathrm{B})$. The functors defined in this way are identified in [3] with the Hecke functors in the geometric Langlands correspondence. For some branes B one may represent for each fixed $P \in C$ the resulting modification as the tensor product of $\mathcal{H}(\mathrm{B})$ with a finite-dimensional representation $V$ of ${ }^{L} G$. One says that the brane B satisfies the Hecke eigenvalue property if the family of modifications obtained by varying the point $P \in C$ glues into a local system.

A family of branes $F_{\mu}$ is identified in [3] having this property. The branes $F_{\mu}$ are supported on fibers of the Hitchin's torus fibration. S-duality of $N=4 \mathrm{SYM}$ gets represented within the sigma model with target $\mathcal{M}_{H}(G)$ as a variant of SYZ mirror symmetry, relating the branes $\mathrm{F}_{\mu}$ to branes the dual sigma model with target $\mathcal{M}_{H}\left({ }^{L} G\right)$ represented by skyscraper sheaves $\check{\mathrm{F}}_{\mu}$ having pointlike support at $\mu \in \mathcal{M}_{H}\left({ }^{L} G\right)$.

\subsection{AGT-correspondence - approach of Nekrasov-Witten}

Alday, Gaiotto and Tachikawa discovered a relation between the instanton partition functions of certain $N=2$ supersymmetric gauge theories and conformal blocks of the Virasoro algebra [1]. This discovery has stimulated a lot of work leading in particular to various generalisations of such relations. In an attempt to explain the relations discovered in [1], Nekrasov and Witten 
considered four-dimensional $N=2$ supersymmetric gauge theories of class $\mathcal{S}$ obtained from the maximally supersymmetric six-dimensional QFT on manifolds of the form $\mathcal{M}^{4} \times C$ by compactification on the Riemann surface $C$. For the case associated to the Lie algebra $\mathfrak{g}=$ $\mathfrak{s l}_{2}$ one has weakly coupled Lagrangian descriptions of the resulting theory associated to the choice of a pants decomposition $\sigma$ of $C$. For four-manifolds $\mathcal{M}^{4}$ which can be represented as a fibered product locally of the form $\mathbb{R} \times I \times S^{1} \times S^{1}$ it is argued in [4] that (i) an $\Omega$ deformation with parameters $\epsilon_{1}, \epsilon_{2}$ can be defined, and (ii) an effective representation is obtained by compactification on $S^{1} \times S^{1}$ in terms of a sigma-model with target $\mathcal{M}_{H}(G)$ on $R \times I$. The coupling parameter of this sigma model is $\epsilon_{1} / \epsilon_{2}$.

The end points of the interval $I$ in the representation $\mathcal{M}^{4} \simeq \mathbb{R} \times I \times S^{1} \times S^{1}$ correspond to points where $\mathcal{M}^{4}$ is perfectly regular. One must therefore have distinguished boundary condition in the sigma-model with target $\mathcal{M}_{H}(G)$ on $R \times I$ describing the compactification of a class $\mathcal{S}$ theory on $\mathcal{M}^{4}$. When the compactification yields a sigma model with target $\mathcal{M}_{H}(G)$, it is argued in [4] that the corresponding boundary conditions are described by a variant $\mathrm{B}_{\mathrm{cc}}$ of the canonical coisotropic brane at $\mathbb{R} \times\{0\}$, and a new type of brane called the "brane of opers", here denoted by $B_{o p}$, respectively 1$]$ The brane $B_{o p}$ is the mirror dual of $B_{c c}$, and it is proposed in [4] that the brane $\mathrm{B}_{\mathrm{op}}$ is a Lagrangian brane supported on the variety of opers within $M_{H}(G)$.

In [4] it is furthermore proposed that the space $\mathcal{H}=\operatorname{Hom}_{\mathcal{M}_{H}(G)}\left(\mathrm{B}_{\mathrm{cc}}, \mathrm{B}_{\mathrm{op}}\right)$ can be identified with the space of Virasoro conformal blocks. In order to motivate this identification, Nekrasov and Witten note that the algebra $\mathcal{A}_{\mathrm{cc}}^{\hbar}(G)=\operatorname{Hom}_{\mathcal{M}_{H}(G)}\left(\mathrm{B}_{\mathrm{cc}}, \mathrm{B}_{\mathrm{cc}}\right)$ with $\hbar=\epsilon_{1} / \epsilon_{2}$ is isomorphic to the algebra of Verlinde line operators acting on the space of Virasoro conformal blocks. Mirror symmetry produces a dual description of $\mathcal{H}(G) \simeq \mathcal{H}\left({ }^{\mathrm{L}} G\right)$ as the space $\operatorname{Hom}_{\mathcal{M}_{H}\left({ }^{L} G\right)}\left(\mathrm{B}_{\mathrm{op}}^{\prime}, \mathrm{B}_{\mathrm{cc}}^{\prime}\right)$, with $B_{o p}^{\prime}$ and $B_{c c}^{\prime}$ being close relatives of $B_{o p}$ and $B_{c c}$, respectively, with modified SUSY invariance properties. In the dual representation one has an obvious right action of the algebra $\check{\mathcal{A}}_{\mathrm{cc}}^{1 / \hbar}\left({ }^{L} G\right)=\operatorname{Hom}_{\mathcal{M}_{H}\left({ }^{L} G\right)}\left(\mathrm{B}_{\mathrm{cc}}^{\prime}, \mathrm{B}_{\mathrm{cc}}^{\prime}\right)$ with action defined by "joining open strings" on the right boundary of the strip $I$. The existence of (almost) commuting actions of $\mathcal{A}_{\mathrm{cc}}^{\hbar}(G)$ and $\check{\mathcal{A}}_{\mathrm{cc}}^{1 / \hbar}\left({ }^{L} G\right)$ is a characteristic feature of the space of Virasoro conformal blocks.

\subsection{The other way around}

It is no accident that the work of Nekrasov and Witten [4] has many elements in common with the approach Kapustin and Witten [3]. A common root can be found in the fact that both the class $\mathcal{S}$-theories and $N=4 \mathrm{SYM}$ [15] can be obtained as compactifications of the sixdimensional $(2,0)$-theory on six-manifolds $\mathcal{M}^{6}=\mathcal{M}^{4} \times C$, where $C$ is a Riemann surface, and

\footnotetext{
${ }^{1}$ The branes denoted $B_{\mathrm{cc}}$ in this context are similar but not identical with the brane considered in [3]. The paper [4] used the notation $B_{N^{\prime}}$ for the brane denoted $\mathrm{B}_{\mathrm{op}}$ here
} 
$\mathcal{M}^{4}$ is a four-manifold locally represented as a circle fibration locally of the form $\mathbb{R} \times I \times S^{1} \times S^{1}$. Compactification on $C$ yields class $\mathcal{S}$-theories [16], while compactification on $S^{1} \times S^{1}$ yields $N=4 \mathrm{SYM}$ on $\mathbb{R} \times I \times C$, the set-up considered in [3] as was further discussed in [15].

One should note, however, that different topological twists are used in the two compactifications, making the comparison of the results somewhat subtle. This fact can nevertheless be used to relate supersymmetric boundary conditions in the $2 \mathrm{~d}$ sigma model with target $\mathcal{M}_{H}$ arising from compactification of class $\mathcal{S}$-theories to boundary conditions in $N=4 \mathrm{SYM}$ on $C$. These boundary conditions have been classified in the work of Gaiotto and Witten [17]. In this way, one may establish a relationship between the canonical coisotropic brane and pure Neumann boundary conditions in $N=4 \mathrm{SYM}$ [3, 8]. Exchanging the two circles in $S^{1} \times S^{1}$ gets related to the S-duality of $N=4 \mathrm{SYM}$ which implies relations between its boundary conditions studied in [18]. This led [8] to relate the brane $B_{o p}$, the mirror dual of the canonical coisotropic brane in [4], to the boundary condition descending from the so-called Nahm pole boundary conditions in $N=4 \mathrm{SYM}$, as will briefly be discussed in Subsection 3.2 below.

\subsection{Other approaches}

As noted above, one needs to use different twists in the two reductions from six to four dimensions considered above. In order to get the set-up studied in [4], for example, one needs to twist the $(2,0)$ theory on $\mathcal{M}^{4} \times C$ in such a way that it becomes topological on $\mathcal{M}^{4}$. A different twist is obviously needed to get the topologically twisted $N=4 \mathrm{SYM}$ on $\mathbb{R} \times I \times C$ studied in [3] from the six-dimensional $(2,0)$ theory.

In order to describe the dimensional reduction of topologically twisted $N=4 \mathrm{SYM}$ on $\mathbb{R} \times$ $I \times C$ one may find it natural to consider boundary conditions that are purely topological, not depending on the complex structure on $C$. This point of view motivated Ben-Zvi and Nadler [19] to propose the Betti geometric Langlands conjecture as a purely topological variant of the geometric Langlands correspondence formulated in [11] that is naturally adapted to the fourdimensional TQFT's studied in [3].

Yet another approach towards understanding the AGT-correspondence was proposed by Cordova and Jafferis in [20]. It starts from six-manifolds of the form $\mathcal{M}^{4} \times C$, with $\mathcal{M}^{4}$ being a squashed four-sphere, represented as three-sphere fibration over an interval $I_{0}$, locally $\mathcal{M}^{4} \sim I_{0} \times S_{\epsilon_{1} \epsilon_{2}}^{3}$ with $\epsilon_{1}, \epsilon_{2}$ now being the squashing parameters. Considering partial topological twisting on $C$, it is argued that the consequence of Weyl invariance of the $(2,0)$ theory with the twist on $C$ is the fact that partition functions depend neither on the overall radius of $\mathcal{M}^{4}$ nor on the volume of $C$. The effective description at small volume of $C$ will be given by the partition functions of class $S$ theories on (squashed) four-spheres studied in the work of Pes- 
tun [21] and many subsequent publications. It is argued that an equivalent description in terms of a CFT on $C$ is obtained by first compactifying on the three spheres $S_{\epsilon_{1} \epsilon_{2}}^{3}$ appearing in the representation $\mathcal{M}^{4} \sim I_{0} \times S_{\epsilon_{1} \epsilon_{2}}^{3}$. Using five-dimensional SYM theory as an intermediate step, Cordova and Jafferis found an effective description in terms of complex Chern-Simons theory on $I_{0} \times C$. The boundary conditions at the ends of $C$ are determined by the Nahm pole boundary conditions studied in [17, 18, 8]. A generalised version of the usual correspondence between Chern-Simons theory and two-dimensional conformal field theory can then be invoked, with Nahm pole boundary conditions translating into the constraints reducing the WZNW models to the conformal Toda theories.

\section{Defects of co-dimension two and surface operators}

$M$ theory suggests a natural extension of the set-up considered above. Viewing the sixdimensional $(2,0)$ theory as the effective theory on a stack of M5-branes in M-theory leads to a natural extension of the set-up by an additional stack of M5'-branes sharing part of the support with the original M5-branes, as indicated in the following table:

\begin{tabular}{l||c|c|c|c|c|c|c|c|c|c|c} 
Brane & 0 & 1 & 2 & 3 & 4 & 5 & 6 & 7 & 8 & 9 & 10 \\
\hline \hline M5 & $\mathrm{x}$ & $\mathrm{x}$ & $\mathrm{x}$ & $\mathrm{x}$ & & & $\mathrm{x}$ & & & & $\mathrm{x}$ \\
M5, & $\mathrm{x}$ & $\mathrm{x}$ & & & & & $\mathrm{x}$ & & $\mathrm{x}$ & $\mathrm{x}$ & $\mathrm{x}$
\end{tabular}

Depending on the type of compactification considered one gets surface operators in class $\mathcal{S}$ theories, or line operators in $N=4$ SYM theory. This will be the first type of modification turning out to be crucial for our story.

\subsection{Surface operators in class $\mathcal{S}$ theories}

By now there exists a fair amount of evidence that an effective description of a system of M5-M5'-branes obtained by compactification on $C$ is provided by theories of class $\mathcal{S}$ modified by the presence of a certain type of surface operator. The relevant surface operators can be described by prescribing a certain type of singular behaviour of the gauge fields along a two-dimensional submanifold $\mathcal{M}^{2} \subset \mathcal{M}^{4}$ characterised by a set of parameters $x=\left(x_{1}, \ldots, x_{3 g-3+n}\right)$ if $C$ has genus $g$ and $n$ punctures [22, 23]. A generalisation $\mathcal{Z}_{\sigma}^{\text {inst }}\left(a ; x, \tau ; \epsilon_{1}, \epsilon_{2}\right)$ of the instanton partition functions can be defined in the presence of such surface operators, carrying an additional dependence on the parameters $x$ on top of the dependencies on the scalar zero mode values $a$, the UV gauge couplings associated to complex structure moduli $\tau$ of $C$, and the parameters $\epsilon_{1}, \epsilon_{2}$ associated to the Omega-deformation. 
The subscript $\sigma$ refers to the pants decomposition determining the Lagrangian representation being used. Explicit calculations in [24, 22, 25, 26, 27] gave evidence for a generalisation of the AGT-correspondence, identifying $\mathcal{Z}_{\sigma}^{\text {inst }}\left(a ; x, \tau ; \epsilon_{1}, \epsilon_{2}\right)$ with the chiral partition functions $\mathcal{Z}_{\sigma}^{\mathrm{WZW}}(a ; x, \tau ; k)$ associated to conformal blocks of the affine Lie algebras $\hat{\mathfrak{g}}_{k}$ at level $k=-h^{\vee}-\epsilon_{2} / \epsilon_{1}$ defined by the gluing construction. The parameters $x$ get identified with coordinates on the moduli space $\operatorname{Bun}_{G}$ of holomorphic $G$-bundles on $C$ under this correspondence. A proof of this generalisation of the AGT-correspondence will follow from the result announced in [2] that $\mathcal{Z}_{\sigma}^{\text {inst }}$ satisfies the KZB equations 2

Our goal in this section will be to generalise the approach of [4, 8] to theories of class $\mathcal{S}$ in the presence of surface operators of co-dimension two. We will propose an effective description in terms of the two-dimensional sigma model with target $\mathcal{M}_{H}(G)$ in which the brane of opers $\mathrm{B}_{\mathrm{op}}$ gets replaced by a family of Lagrangian branes $\mathrm{L}_{x}^{(2)}$ supported on the fibers of Hitchin's second fibration over bundles $\mathcal{E}_{x}$ labelled by coordinate $x$ for $\mathrm{Bun}_{G}$. The relevance of the branes $\mathrm{L}_{x}^{(2)}$ in this context and the relation to conformal blocks has first been proposed by E. Frenkel in [28], as has been pointed out to one of us (J.T.) in 2012. Our goal in the following will be to offer additional support for this proposal.

\subsection{Nahm pole boundary conditions}

Gaiotto and Witten have classified 1/2 BPS boundary conditions of $N=4$ SYM in [17] using the data $(\rho, H, T)$, where $\rho: \mathfrak{s l}_{2} \rightarrow \mathfrak{g}$ is an embedding of $\mathfrak{s l}_{2}$ into the Lie algebra $\mathfrak{g}$ of the gauge group $G_{c}, H$ is a subgroup of the commutant in $G_{c}$ of the image of $\rho$, and $T$ is a threedimensional SCFT with $N=4$ supersymmetry and at least $H$ global symmetry. We will only need two of the simplest of these boundary conditions. In the following we will first briefly review the so-called Nahm pole boundary condition studied in [8] which is associated to a triple $\left(\rho\right.$, Id, $\left.T_{\emptyset}\right)$, where $\rho$ is a principal $\mathfrak{s l}_{2}$-embedding, and $T_{\emptyset}$ stands for the trivial three-dimensional SCFT. We will then discuss the even simpler case where $\rho$ is replaced by the trivial embedding mapping $\mathfrak{s l}_{2}$ to $0 \in \mathfrak{g}$, which will be of particular interest for us.

It is for our purposes sufficient to describe the Nahm pole boundary conditions for the solutions of the BPS-equations [3] characterising field configuration in $N=4$ SYM preserving certain supersymmetries. Restricting attention to solutions to the BPS-equations on $\mathbb{R} \times \mathbb{R}_{+} \times C$ which

\footnotetext{
${ }^{2}$ see also [26] for an earlier result in this direction.
} 
are invariant under translations along $\mathbb{R}$, one gets a system of differential equations of the form

$$
\begin{aligned}
& {\left[\mathcal{D}_{z}, \mathcal{D}_{\bar{z}}\right]=0, \quad\left[\mathcal{D}_{y}, \mathcal{D}_{z}\right]=0, \quad\left[\mathcal{D}_{y}, \mathcal{D}_{\bar{z}}\right]=0} \\
& \sum_{i=1}^{3}\left[\mathcal{D}_{i}, \mathcal{D}_{i}^{\dagger}\right]=0
\end{aligned}
$$

where the notations $z=x_{2}+i x_{3}$ and $y=x_{1}$ have been used, and the differential operators $\mathcal{D}_{i}$ are of the form 3

$$
\begin{array}{ll}
\mathcal{D}_{z}=\zeta \partial_{z}+\mathcal{A}_{z}, & \mathcal{A}_{z}=\zeta A_{z}+\phi_{z}, \\
\mathcal{D}_{\bar{z}}=\partial_{\bar{z}}+\mathcal{A}_{\bar{z}}, & \mathcal{A}_{\bar{z}}=A_{\bar{z}}+\zeta \phi_{\bar{z}},
\end{array} \quad \mathcal{D}_{y}=\partial_{y}+A_{y}-i \phi_{y} .
$$

The parameter $\zeta$ determines the supersymmetries that are preserved. It is proposed in [8] that the space of solutions to (3.1) modulo compact gauge transformations is isomorphic to the moduli space of the solutions to the "F-term" equations (3.1a) modulo complex gauge transformations. Equations $\left[\mathcal{D}_{z}, \mathcal{D}_{\bar{z}}\right]=0$ determine a flat complex connection on $C$ at each fixed $y$. The remaining equations in (3.1a) imply that the $y$-dependence of this flat connection is represented by complex gauge transformations.

Boundary conditions of Nahm pole type are defined in [8] by demanding that the solutions to (3.1) have a singular behaviour of the form

$$
\mathcal{A}_{z} \underset{y \rightarrow 0}{\sim} \mathfrak{t}_{-} y^{-1}+\mathcal{O}\left(y^{0}\right), \quad \mathcal{A}_{\bar{z}} \underset{y \rightarrow 0}{\sim} \mathcal{O}\left(y^{0}\right), \quad \mathcal{A}_{1} \underset{y \rightarrow 0}{\sim} \mathfrak{t}_{3} y^{-1}+\mathcal{O}\left(y^{0}\right),
$$

with $\mathfrak{t}_{+}=\mathfrak{t}_{1}+i \mathfrak{t}_{2}$, and $\mathfrak{t}_{i}, i=1,2,3$, being the generators of a principal $\mathfrak{s l}_{2}$ subalgebra of $\mathfrak{g}$. By a gauge transformation we may always set $\mathcal{A}_{\bar{z}}$ to zero, allowing us to represent the flat connection on $C$ we get at each $y$ as a local system $\left(\mathcal{E}_{y}, \nabla_{y}^{\prime}\right)$ consisting of a holomorphic bundle and a holomorphic connection $\nabla_{y}^{\prime}=d z\left(\partial_{z}+\mathcal{A}_{z}(z ; y)\right)$. In the case $\mathfrak{g}=\mathfrak{s l}_{2}$, we may reformulate the first condition in (3.3) as the condition that there exists a basis of sections $s=\left\{s_{1}, s_{2}\right\}$ with respect to which $\mathcal{A}$ has the form $\mathcal{A}=g \tilde{\mathcal{A}} g^{-1}+g d g^{-1}$, with

$$
\tilde{\mathcal{A}}_{z} \underset{y \rightarrow 0}{\sim}\left(\begin{array}{ll}
0 & t \\
1 & 0
\end{array}\right)+\mathcal{O}\left(y^{1}\right), \quad g \underset{y \rightarrow 0}{\sim}\left(\begin{array}{cc}
y^{1 / 2} & 0 \\
0 & y^{-1 / 2}
\end{array}\right)+\mathcal{O}\left(y^{0}\right) .
$$

Horizontal sections $(d+\mathcal{A}) s=0$ will then have a first component $s_{1}$ vanishing as $y^{1 / 2}$. As explained in [8], this implies that the local system $\lim _{y \rightarrow 0}\left(\tilde{\mathcal{E}}_{y}, \tilde{\nabla}_{y}^{\prime}\right)$ on $C$ is an oper.

The Nahm pole boundary condition has the feature that it breaks $G_{c}$ maximally since the commutant of the principal $\mathfrak{s l}_{2}$-embedding is trivial. At the opposite extreme, associated to the

\footnotetext{
${ }^{3}$ Our conventions differ slightly from [8].
} 
trivial $\mathfrak{s l}_{2}$-embedding, one gets a similar boundary condition associated to a triple $\left(0, \operatorname{Id}, T_{\emptyset}\right)$ by fixing the boundary value of the gauge field $\mathcal{A}_{\bar{z}}$.

In the reduction to two dimensions having fixed $\mathcal{A}_{\bar{z}}$ at the boundary of $I$ implies having fixed a holomorphic bundle on $C$, leaving the $(1,0)$ part of the complex gauge field unconstrained. The moduli space $\mathcal{M}_{d R}(G)$ of pairs $\left(\mathcal{E}, \nabla_{\zeta}^{\prime}\right)$ is isomorphic to the Hitchin moduli space $\mathcal{M}_{H}(G)$ via the non-abelian Hodge $(\mathrm{NAH})$ correspondence $^{(g)}$. Fixing $\mathcal{E}$ therefore defines a submanifold in $\mathcal{M}_{H}(G)$ which is Lagrangian with respect to the holomorphic symplectic form $\Omega_{\zeta}$, and holomorphic w.r.t. to the complex structure $I_{\zeta}$. For $\zeta=i$ one has $\Omega_{\zeta}=\Omega_{J}, I_{\zeta}=J$, leading to the identification of the brane coming from the reduction of the zero Nahm pole boundary condition as an (A,B,A)-brane in the A-model with the symplectic structure $\omega_{I}$ used in [4].

We are proposing that the zero Nahm pole boundary condition represents the presence of a surface operator of co-dimension two. Indeed, as was argued in [23], the presence of a codimension two surface operator naturally introduces additional background data which can be geometrically represented as the choice of a holomorphic bundle on $C$.

\subsection{Relation to conformal blocks}

The Lagrangian submanifolds $\mathrm{L}_{\mathcal{E}, \zeta}^{(2)}$ of $\mathcal{M}_{H}(G)$ defined by considering pairs $\left(\mathcal{E}, \nabla^{\prime}\right)$ with fixed $\mathcal{E}$ are sometimes called the fibers of Hitchin's second fibration. Picking a system $x$ of coordinates for $\operatorname{Bun}_{G}$ we will use the notation $\mathrm{L}_{x}^{(2)}$ for $\mathrm{L}_{\mathcal{E}_{x}, i}^{(2)}$ with $\mathcal{E}_{x}$ being a bundle representing the point in $\operatorname{Bun}_{G}$ specified by the coordinates $x$.

The results on the generalisation of the AGT-correspondence in the presence of the surface operators of co-dimension two mentioned above suggest that the space $\mathcal{H}_{x}^{(2)}=\operatorname{Hom}_{\mathcal{M}_{H}(G)}\left(\mathcal{B}_{\mathrm{cc}}, \mathrm{L}_{x}^{(2)}\right)$ can be identified with the space of conformal blocks of the affine Lie algebra $\hat{\mathfrak{g}}_{k}$ at level $k=-h^{\vee}-\frac{\epsilon_{2}}{\epsilon_{1}}$ on $C$.

We need to note, however, that finding the proper definition of both $\mathcal{H}_{x}^{(2)}$ and the relevant spaces of conformal blocks is nontrivial in the infinite-dimensional situation at hand. The usual algebraic definition of conformal blocks defines a space $\mathcal{C} \mathcal{B}_{\mathrm{al}}\left(C, \hat{\mathfrak{g}}_{k}, \mathcal{E}_{x}\right)$ that is too large to be relevant for us. We'll need to consider a subspace denoted $\mathcal{C} \mathcal{B}_{\text {te }}\left(C, \hat{\mathfrak{g}}_{k}, \mathcal{E}_{x}\right)$ of "tempered" conformal blocks. The relation between these two spaces is in some respects similar to the relation between spaces $\mathcal{K}_{\text {al }}$ of formal power series $\sum_{n \in \mathbb{Z}} a_{n} z^{n}$ to the spaces $\mathcal{K}_{\text {te }}$ of tempered distributions on the unit circle. The latter can be represented by Fourier series $\sum_{n \in \mathbb{Z}} a_{n} e^{i n \sigma}$ in the distributional sense, leading to an embedding of $\mathcal{K}_{\text {te }}$ into $\mathcal{K}_{\text {al. }}$. However, being tempered imposes growth conditions on the coefficients $a_{n}$, making $\mathcal{K}_{\text {te }}$ strictly smaller than $\mathcal{K}_{\text {al }}$.

An algebraic counterpart for $\mathcal{H}_{x}^{(2)}$, here denoted $\mathcal{H}_{\mathrm{al}, x}^{(2)}$, was proposed in the work [28] of E. 
Frenkel, where it was proven that $\mathcal{H}_{\mathrm{al}, x}^{(2)} \simeq \mathcal{C} \mathcal{B}_{\mathrm{al}}\left(C, \hat{\mathfrak{g}}_{k}, \mathcal{E}_{x}\right)$. We will argue that a different definition for $\mathcal{H}_{x}^{(2)}$ is appropriate in this context, leading to an isomorphism $\mathcal{H}_{x}^{(2)} \simeq \mathcal{C B}_{\text {te }}\left(C, \hat{\mathfrak{g}}_{k}, \mathcal{E}_{x}\right)$ with a subspace $\mathcal{C} \mathcal{B}_{\text {te }}\left(C, \hat{\mathfrak{g}}_{k}, \mathcal{E}_{x}\right)$ of "tempered" conformal blocks within $\mathcal{C} \mathcal{B}_{\text {al }}\left(C, \hat{\mathfrak{g}}_{k}, \mathcal{E}_{x}\right)$. We can not discuss the definition of $\mathcal{C B}_{\text {te }}\left(C, \hat{\mathfrak{g}}_{k}, \mathcal{E}_{x}\right)$ fully in this short note, we plan to return to this point elsewhere. Instead we will in the following discuss evidence for $\mathcal{H}_{x}^{(2)} \simeq \mathcal{C B}_{\text {te }}\left(C, \hat{\mathfrak{g}}_{k}, \mathcal{E}_{x}\right)$ coming from the $2 d$ sigma model.

\subsubsection{Module structures}

To begin with, let us note that $\mathcal{H}_{x}^{(2)}$ has two natural module structures coming from the vertex operators associated to the elements of the algebra $\mathcal{A}_{\mathrm{cc}}^{\hbar}=\operatorname{Hom}_{\mathcal{M}_{G}(C)}\left(\mathrm{B}_{\mathrm{cc}}, \mathrm{B}_{c c}\right)$. For the case of interest one should represent $\mathcal{M}_{H}(G)$ as moduli space of flat complex connections on $C$ using the NAH correspondence, allowing us to consider two algebraic structures coming from the representation of $\mathcal{M}_{H}(G)$ as character variety ${ }^{(g)} \mathcal{M}_{B}(G)$ and as moduli space $\mathcal{M}_{d R}(G)$ of pairs $\left(\mathcal{E}, \nabla^{\prime}\right)$, respectively. Different algebraic structures determine different sets of basic field variables to be used in the definition of the vertex operators in $\mathcal{A}_{\mathrm{cc}}$. One may accordingly distinguish $\mathcal{A}_{B}^{\hbar}=\operatorname{Hom}_{\mathcal{M}_{B}(C)}\left(\mathrm{B}_{\mathrm{cc}}, \mathrm{B}_{c c}\right)$ and $\mathcal{A}_{d R}^{\hbar}=\operatorname{Hom}_{\mathcal{M}_{d R}(C)}\left(\mathrm{B}_{\mathrm{cc}}, \mathrm{B}_{c c}\right)$, where $\hbar=\epsilon_{1} / \epsilon_{2}$.

On the one hand it was argued in [4] that the algebra $\mathcal{A}_{B}^{\hbar}$ can be identified with the quantised algebra of functions on $\mathcal{M}_{B}(G)$. The arguments in [29] may, on the other hand, be applied to the situation studied in [4] leading to the conclusion that $\mathcal{A}_{d R}^{\hbar} \simeq \mathcal{D}_{\hbar}$, the quantised algebra of functions on $\mathcal{M}_{d R}(G)$, which may be identified with the algebra of differential operators on a certain line bundle $\mathcal{L}^{-h^{\vee}-\epsilon_{2} / \epsilon_{1}}$ over $\operatorname{Bun}_{G}$.

It well-known that the space $\mathcal{C B}_{\text {al }}\left(C, \hat{\mathfrak{g}}_{k}, \mathcal{E}_{x}\right)$ of conformal blocks of the affine Lie algebra $\hat{\mathfrak{g}}_{k}$ at level $k=-h^{\vee}-\frac{\epsilon_{2}}{\epsilon_{1}}$ has a module structure with respect to $\mathcal{A}_{d R}^{\hbar}$. The $\mathcal{A}_{d R}^{\hbar}$-module structure is a direct consequence of the defining Ward identities.

A $\mathcal{A}_{B}^{\hbar}$-module structure can defined on spaces of conformal blocks by using degenerate representations of $\hat{\mathfrak{g}}_{k}$ to define analogs of the Verlinde line operators [30, 31] in this case. It will be shown elsewhere that the algebra of Verlinde line operators on affine Lie algebra conformal blocks may be identified with $\mathcal{A}_{B}^{\hbar}$. The known definitions work with conformal blocks constructed using the gluing construction. Such conformal blocks have particularly nice properties, one of which being that the canonical connection may be integrated over all of $\operatorname{Bun}_{G}^{\mathrm{vs}}$, the subset of $\operatorname{Bun}_{G}$ containing the "very stable" bundles not admitting a nilpotent Higgs field which is not the case for generic elements of $\mathcal{C B}^{\text {al }}\left(C, \hat{\mathfrak{g}}_{k}, \mathcal{E}_{x}\right)$. This suggests that the $\mathcal{A}_{B}^{\hbar}$-module structure can only be defined on suitable subspaces $\mathcal{C B}_{\text {te }}\left(C, \hat{\mathfrak{g}}_{k}, \mathcal{E}_{x}\right)$ of "tempered" conformal blocks.

The existence of two module structures on $\mathcal{H}_{x}^{(2)}$ can be regarded as a first piece of evidence for the conjectured isomorphism $\mathcal{H}_{x}^{(2)} \simeq \mathcal{C B}_{\text {te }}\left(C, \hat{\mathfrak{g}}_{k}, \mathcal{E}_{x}\right)$. Further evidence will be given below. 


\subsubsection{Relation to $\bar{\partial}$-cohomology}

To begin with, let us return to the case studied by Nekrasov and Witten in [4]. We first note that the space $\operatorname{Hom}_{\mathcal{M}_{H}(G)}\left(\mathrm{B}_{\mathrm{cc}}, \mathrm{B}_{\mathrm{op}}\right)$ has a realisation in the A-model with symplectic structure $\omega=\omega_{I}$. The boundary conditions on the strip are given as the canonical coisotropic A-branes $\mathrm{B}_{\mathrm{cc}}$ and the Lagrangian A-brane $\mathrm{B}_{\mathrm{op}}$. The Chan-Paton curvature $F$ is given by the symplectic form $\omega_{J}$ on Hitchin moduli space. It follows that the complex structure determined by the canonical coisotropic A-brane $\mathrm{B}_{\mathrm{cc}}$ is $\omega^{-1} F=K$. It is argued in [4] that in this case the space $\operatorname{Hom}_{\mathcal{M}_{H}(G)}\left(\mathrm{B}_{\mathrm{cc}}, \mathrm{B}_{\mathrm{op}}\right)$ can be identified with the space of holomorphic sections of the line bundle $K_{\mathrm{op}}^{1 / 2}$ on the subspace $\mathrm{Op}_{\mathfrak{s l}_{2}}(C)$ of opers in $\mathcal{M}_{d R}(G)$. Indeed, the space $\operatorname{Hom}_{\mathcal{M}_{H}(G)}\left(\mathrm{B}_{\mathrm{cc}}, \mathrm{B}_{\mathrm{op}}\right)$ will have a realisation as space of functions of the zero modes of the A-model on the strip. We have Neumann-type boundary conditions for the sigma model fields representing coordinates on $\mathrm{Op}_{\mathfrak{s l}_{2}}(C)$ on both ends of the strip, while the remaining fields have Dirichlet-type boundary conditions on one end only. The zero modes of the A-model may therefore be represented in terms of coordinates on $\mathrm{Op}_{\mathfrak{s l}_{2}}(C)$. Standard arguments briefly reviewed in [4] may then be used to identify the space of supersymmetric ground states of the sigma model on the strip with the $\bar{\partial}$-cohomology with values in $K_{\mathrm{op}}^{1 / 2}$.

\subsubsection{Relation to conformal blocks: Virasoro case}

Note that $\mathrm{Op}_{\mathfrak{s l}_{2}}(C)$ is (non-canonically) isomorphic to the complex vector space $H^{0}\left(C, K_{C}^{2}\right)$ which is topologically trivial. The space $H^{0}\left(C, K_{C}^{2}\right)$ is canonically isomorphic to the cotangent fiber $\left.T^{*} \mathcal{T}(C)\right|_{C}$ to the Teichmüller space $\mathcal{T}(C)$. It will be important for us to observe that there is a map from the cotangent space $\left.T^{*} \mathcal{T}(C)\right|_{C}$ to the Teichmüller space $\mathcal{T}(C)$ relating the natural complex structures on these spaces.

In order to introduce the complex structure on $\mathcal{T}(C)$ one may describe the Teichmüller variations in terms of the harmonic Beltrami differentials, which are $(-1,1)$-forms $\mu$ satisfying $\partial_{z}(\eta \mu)=0$, where $\eta$ is the hyperbolic metric uniquely determined by the complex structure on $C$. The complex structure on the vector space of harmonic Beltrami differentials thereby defines the complex structure on $\mathcal{T}(C)$. To a holomorphic $(2,0)$-form $\theta$ one may assign the corresponding harmonic Beltrami differential $\eta^{-1} \bar{\theta}$. This defines a complex anti-linear map from $H^{0}\left(C, K_{C}^{2}\right)$ to the space of harmonic Beltrami differentials expressing the relation be-

tween the natural complex structures on $\mathcal{T}(C)$ and on $H^{0}\left(C, K_{C}^{2}\right)$, respectively. It allows us to identify the space of holomorphic functions on $\mathrm{Op}_{\mathfrak{s l}_{2}}(C)$ with the space $\operatorname{Fun}_{\text {hol }}(\mathcal{T}(C))$ of (anti-) holomorphic functions on $\mathcal{T}(C)$.

The spaces $\operatorname{Fun}_{\text {hol }}(\mathcal{T}(C))$, on the other hand, are known to be isomorphic with (sub-)spaces 
of Virasoro conformal blocks. Conformal blocks on closed 4 surfaces $C$ are defined as linear functionals $f$ on the vacuum representation $V_{0}$ of the Virasoro algebra satisfying the conformal Ward identities describing invariance of $f$ under the natural action of the algebra of vector fields holomorphic away from a point. The vector space of conformal blocks carries a canonical projectively flat connection defined by means to the Virasoro action on $V_{0}$. The curvature of the canonical connection may be trivialised locally on the moduli space $\mathcal{M}_{g}$ of complex structures. On suitable subspaces of the space of all algebraically defined conformal blocks one may integrate the resulting flat connection to define horizontal sections $f_{\tau}$ on open subsets of $\mathcal{M}_{g}$ with local coordinates $\tau$. The values of the conformal blocks $\mathcal{Z}_{f}(\tau)=f_{\tau}\left(v_{0}\right)$ on the highest weight vector $v_{0}$ are called chiral partition functions. The Virasoro uniformization theorem and conformal Ward identities relate the derivatives of $\mathcal{Z}_{f}(\tau)$ to the data characterising the conformal blocks $f$. One thereby gets a one-to-one correspondence between (locally defined) functions $\mathcal{Z}(\tau)$ and "integrable" conformal blocks $f$. This correspondence is essentially canonical: The Virasoro uniformisation describes the local structure of $\mathcal{M}_{g}$, and this is encoded in the definition of the conformal blocks.

\subsubsection{Relation to conformal blocks: Kac-Moody case}

It is possible to argue that $\mathcal{H}_{x}^{(2)}$ is isomorphic to the space $\mathcal{C B}\left(C, \hat{\mathfrak{g}}_{k}, \mathcal{E}_{x}\right)$ of conformal blocks for $\hat{\mathfrak{g}}_{k}$ twisted by the bundle $\mathcal{E}_{x}$ in a similar way as was argued in Section 3.3.3. Following the the same reasoning as described there one would arrive at the conclusion that $\mathcal{H}_{\mathcal{E}}$ is isomorphic to the space of holomorphic functions on $H^{0}\left(C, \operatorname{End}(\mathcal{E}) \otimes K_{C}\right)$. The space $H^{0}(C, \operatorname{End}(\mathcal{E}) \otimes$ $\left.K_{C}\right)$ is the holomorphic cotangent space $T^{*} \operatorname{Bun}_{G}$. There exists a canonical hermitian metric on $\operatorname{TBun}_{G}$ which is analogous to the Weil-Petersson metric in Teichmüller theory, defined using the Narasimhan-Seshadri theorem as analog of the uniformisation theorem. This metric identifies the holomorphic co-tangent space with the anti-holomorphic tangent space, relating the natural complex structures on $H^{0}\left(C, \operatorname{End}(\mathcal{E}) \otimes K_{C}\right)$ and $T \operatorname{Bun}_{G}$.

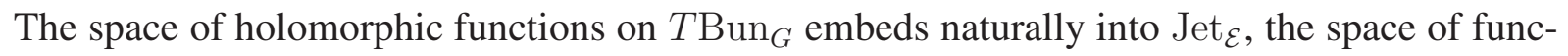
tions on a formal neighbourhood of $\mathcal{E}$ in $\operatorname{Bun}_{G}$. It remains to notice that Jet $t_{\mathcal{E}}$ is isomorphic to the space of conformal blocks, $\operatorname{Jet}_{\mathcal{E}} \simeq \mathcal{C} \mathcal{B}_{\text {al }}\left(C, \hat{\mathfrak{g}}_{k}, \mathcal{E}_{x}\right)$ [32, Section 18.2]. This observation was used by E. Frenkel in [28] to prove the algebraic counterpart $\mathcal{H}_{\mathrm{al}, x}^{(2)} \simeq \mathcal{C B}_{\mathrm{al}}\left(C, \hat{\mathfrak{g}}_{k}, \mathcal{E}_{x}\right)$ of our claim. It follows that spaces of holomorphic functions on $T \operatorname{Bun}_{G}$ can be identified with subspaces of "well-behaved" conformal blocks within $\mathcal{C B}\left(C, \hat{\mathfrak{g}}_{k}, \mathcal{E}_{x}\right)$, further supporting the conjecture $\mathcal{H}_{x}^{(2)} \simeq \mathcal{C B}_{\text {te }}\left(C, \hat{\mathfrak{g}}_{k}, \mathcal{E}_{x}\right)$ proposed above.

\footnotetext{
${ }^{4}$ We temporarily restrict to this case to simplify the exposition.
} 


\section{Partition functions versus conformal blocks}

Partition functions play a central role in the AGT-correspondence and its generalisations, while they do not appear in the approach of Kapustin and Witten to the geometric Langlands correspondence. In order to develop a unifying framework we will now discuss how to define the relevant partition functions in the effective description furnished by the two-dimensional sigma models.

\subsection{TQFT set-up in four and two dimensions}

Using the reduction of class $\mathcal{S}$-theories to the two-dimensions we will in the following motivate a description of the partition functions within the two-dimensional sigma model with target $\mathcal{M}_{H}(G)$. It will be based on yet another type of boundary condition denoted $\mathrm{L}_{a}^{(1)}$. The notation $\mathrm{L}_{a}^{(1)}$ is motivated by a link to Hitchin's first fibration which will be disccussed later.

Following [4] we will consider topologically twisted class $\mathcal{S}$-theories on hemispheres $B_{\epsilon_{1} \epsilon_{2}}^{4}$ with Omega-deformation. The topologically twisted class $\mathcal{S}$-theory associates a vector space $\mathcal{H}_{\text {top }}=Z\left(M_{\epsilon_{1} \epsilon_{2}}^{3}\right)$ to $M_{\epsilon_{1} \epsilon_{2}}^{3}=\partial B_{\epsilon_{1} \epsilon_{2}}^{4}$, here identified with the cohomology of $Q$, the supercharge that can be preserved on $B_{\epsilon_{1} \epsilon_{2}}^{4}$. One may use the path integral over the $4 \mathrm{~d}$ hemisphere $B_{\epsilon_{1} \epsilon_{2}}^{4}$ to define a vector $\Psi \in \mathcal{H}_{\text {top }}$. Wave-functions $\Psi(a)$ of the vector $\Psi$ may be identified with the partition functions $Z\left(B_{\epsilon_{1}, \epsilon_{2}}^{4} ; \mathcal{B}_{a}\right)$ defined by imposing suitable $Q$-invariant boundary conditions $\mathcal{B}_{a}$ labelled by parameters $a$ at $M_{\epsilon_{1} \epsilon_{2}}^{3}$. Such boundary conditions can be identified with the boundary conditions at the infinity of $\mathbb{R}_{\epsilon_{1} \epsilon_{2}}^{4}$ used to define the Nekrasov partition functions, fixing in particular the zero modes of the scalars in the vector multiplets to have values collected in the vector $a=\left(a_{1}, \ldots, a_{3 g-3+n}\right)$. The boundary conditions $\mathcal{B}_{a}$ define a family of boundary states $\beta_{a}$, allowing us to represent $Z\left(B_{\epsilon_{1}, \epsilon_{2}}^{4} ; \mathcal{B}_{a}\right)$ as an overlap $\left\langle\beta_{a}, \Psi\right\rangle$.

In the reduction of the class $\mathcal{S}$-theory to a $2 d$ topological sigma model one should get the following representation of the $4 \mathrm{~d}$ TQFT data introduced above:

- The vector space $\mathcal{H}_{\text {top }} \simeq Z\left(S_{\epsilon_{1}, \epsilon_{2}}^{3}\right) \rightarrow Z(I)$.

- The vector $\Psi=Z\left(B_{\epsilon_{1}, \epsilon_{2}}^{4}\right) \rightarrow Z\left(T_{\epsilon_{1}, \epsilon_{2}}\right) \in \mathcal{H}_{\text {top }}$, where $T_{\epsilon_{1}, \epsilon_{2}}$ is the open triangle with "upper" side removed, topologically equivalent to $\mathbb{R}_{-} \times I$, partially compactified by adding a point at the infinity of $\mathbb{R}_{-}$. The boundary of $T_{\epsilon_{1}, \epsilon_{2}}$ is $\{0\} \times I$.

- The partition function $Z\left(B_{\epsilon_{1}, \epsilon_{2}}^{4} ; \mathcal{B}_{a}\right) \rightarrow Z\left(T_{\epsilon_{1}, \epsilon_{2}} ; \mathrm{B}_{a}\right)$ gets associated to a triangle $T_{\epsilon_{1}, \epsilon_{2}}$ with a boundary condition $\mathrm{L}_{a}^{(1)}$ assigned to the upper side $\{0\} \times I . \mathrm{L}_{a}^{(1)}$ is defined from the boundary condition $\mathcal{B}_{a}$ assigned to $M_{\epsilon_{1} \epsilon_{2}}^{3}$ by the reduction to one dimension. 
This means that the instanton partition functions $\mathcal{Z}\left(a ; x ; \tau ; \epsilon_{1}, \epsilon_{2}\right)$ get represented by partition functions of the sigma model on a triangle which has sides coloured by $\left(B_{c c}, L_{a}^{(1)}, L_{x}^{(2)}\right)$.

\section{2 $\mathcal{D}$-modules versus partition functions}

States $\psi$ in quantum theory are abstractly represented by vectors in a Hilbert space $\mathcal{H}$. A concrete representation as space of wave-functions $\psi(x)$ is obtained by introducing a family of elements $\delta_{x}$ of the (hermitian) dual $\mathcal{H}^{\dagger}$ of $\mathcal{H}$ allowing us to represented $\psi(x)$ as $\left\langle\delta_{x}, \psi\right\rangle$, with $\langle.,\rangle:. \mathcal{H}^{\dagger} \times \mathcal{H} \rightarrow \mathbb{C}$ being the natural pairing. In the present context we may interpret the partition functions $\mathcal{Z}=\mathcal{Z}\left(a ; x, \tau ; \epsilon_{1}, \epsilon_{2}\right)$ as wave-functions $\phi_{x}(a)=\left\langle\delta_{a}^{(1)}, \phi_{x}\right\rangle$ of an element $\phi_{x} \in \mathcal{H}_{x}^{(2)}:=\operatorname{Hom}_{\mathcal{M}_{H}(G)}\left(\mathrm{B}_{\mathrm{cc}}, \mathrm{L}_{x}^{(2)}\right)$ created by the sigma model path integral over the semiinfinite strip $\mathbb{R}_{-} \times I$. The "boundary state" $\delta_{a}^{(1)}$ represents the boundary condition $\mathrm{L}_{a}^{(1)}$ at $\{0\} \times I$.

The overlap $\left\langle\delta_{a}^{(2)}, \phi_{x}\right\rangle$ has a representation as a partition function in the topological sigma model on a triangle with three different types of boundary conditions $\left(B_{c c}, L_{x}^{(2)}, L_{a}^{(1)}\right)$ assigned to the three sides. Time-reversal symmetry identifies the complex conjugate $\mathcal{Z}^{*}$ of $\mathcal{Z}$ with a partition function in the topological sigma model on a triangle with boundary conditions $\left(\mathrm{B}_{\mathrm{cc}}, \mathrm{L}_{a}^{(1)}, \mathrm{L}_{x}^{(2)}\right)$ appearing in a different order. $\mathcal{Z}^{*}$ admits a Hamiltonian interpretation as a wave-function $\varphi_{a}(x)=\left\langle\delta_{x}^{(2)}, \varphi_{a}\right\rangle$ of an element $\varphi_{a} \in \mathcal{H}_{x}^{(1)}:=\operatorname{Hom}_{\mathcal{M}_{H}(G)}\left(\mathrm{B}_{\mathrm{cc}}, \mathrm{L}_{a}^{(1)}\right)$ created by the sigma model path integral over the semi-infinite strip $\mathbb{R}_{-} \times I$.

Both spaces $\mathcal{H}_{a}^{(1)}$ and $\mathcal{H}_{x}^{(2)}$ have a $\mathcal{A}_{d R}^{\hbar}$-module structure coming from the natural action of the algebra $\mathcal{A}_{d R}^{\hbar}$ on $\operatorname{Hom}_{\mathcal{M}_{H}(G)}\left(\mathrm{B}_{\mathrm{cc}}, \mathrm{B}\right)$. We have argued above that this module structure coincides with the one on spaces of conformal blocks following from the affine algebra Ward identities. The resulting structure as twisted $\mathcal{D}$-module coincides with the one defined by the KZB equations satisfied by the chiral partition functions corresponding to the conformal blocks of affine Lie algebras. The partition functions $\mathcal{Z}$ are solutions to the KZB equations. The $\mathcal{D}$-modules $\mathcal{H}_{a}^{(1)}$ and $\mathcal{H}_{x}^{(2)}$ can be fully characterised by the corresponding spaces of wave-functions, physically represented as the partition functions $\mathcal{Z}$.

In the limit $\epsilon_{2} \rightarrow 0$ discussed below we will propose that $\mathcal{H}_{a}^{(1)}$ appears in the Kapustin-Witten approach to the geometric Langlands correspondence while $\mathcal{H}_{x}^{(2)}$ appears to be a more natural space to consider in the context of generalisations of the AGT correspondence. The relation between the two Hamiltonian interpretations of the partition function $\mathcal{Z}\left(x, a, \tau ; \epsilon_{1}, \epsilon_{2}\right)$ following from an open TQFT version of modular invariance represents a crucial link between these two stories. 


\subsection{Sigma model description of the brane $L_{a}^{(1)}$}

Let us now propose a description of the boundary condition $\mathrm{L}_{a}^{(1)}$ as a Lagrangian submanifold in $\mathcal{M}_{H}(G)$. For future use we will include the label $\sigma$ referring to the Lagrangian representation of class $\mathcal{S}$ theories used to define the branes $\mathrm{L}_{a}^{(1)}$ into the notation, changing it into $\mathrm{L}_{\sigma, a}^{(1)}$. We propose that the branes $\mathrm{L}_{\sigma, a}^{(1)}$ can be described as the orbits in the character variety $\mathcal{M}_{B}(G)$ of the Hamiltonian flows generated by the collection of length functions $a=\left(a_{1}, \ldots, a_{3 g-3+n}\right)$ representing a Poisson-commuting half of the system of complex Fenchel-Nielsen coordinates ${ }^{g)}$ used in a related context in [5]. The orbits are mapped via holonomy map and the NAH correspondence to the Hitchin moduli space $\mathcal{M}_{H}(G)$. The branes $\mathrm{L}_{\epsilon, a}^{(1) \sigma}$ are natural objects from the point of view of the integrable structure of the character variety $\mathcal{M}_{B}(G)$. Different systems of complex Fenchel-Nielsen coordinates are labelled by pants decompositions $\sigma$.

Our proposal is based on the known relations [33] between Wilson loop observables in class $\mathcal{S}$ theories and co-dimension four defects in the six-dimensional $(2,0)$ theory supported on products of two circles $S_{\mathcal{S}}^{1}$ and $S_{C}^{1}$ in $\mathcal{M}^{4}$ and $C$, respectively. These relations carry over to the case where surface operators are present. Restricting attention to Wilson loops not sharing the support of the surface operators in $\mathcal{M}^{4}$ one may argue as before that the eigenvalues of Wilson loop observables can be expressed in terms of the scalar zero modes $a$ appearing in the arguments of the instanton partition functions $\mathcal{Z}\left(a ; x, \tau ; \epsilon_{1}, \epsilon_{2}\right)$.

One may alternatively adapt the arguments in [30] to the case with additional surface operators of codimension two, which can be based on the results described in Section 6

\section{Another type of surface operators}

One of the key ingredients in the geometric Langlands correspondence is the Hecke eigenvalue property. In order to prepare for the discussion of the Hecke eigenvalue property in the following section we will now discuss another type of surface operators giving us a physical realisation of the Hecke functors.

\subsection{String-theoretical motivation}

From the perspective of the six-dimensional $(2,0)$ theory it seems natural to investigate the effects of modifying the theory by defects of co-dimension four. This defines natural probes of the six-dimensional $(2,0)$ theories and their dimensional reductions which have already been investigated extensively in the context of the AGT correspondence [30]. We will now discuss briefly how the picture gets modified if one considers surface operators of co-dimensions two and four 
at the same time. The existence of such systems is suggested by string theory. Following the discussion in [23] one may, in particular, consider a configuration of M5-M5'-M2-branes in M-theory having support indicated in the following table:

\begin{tabular}{l||c|c|c|c|c|c|c|c|c|c|c} 
Brane & 0 & 1 & 2 & 3 & 4 & 5 & 6 & 7 & 8 & 9 & 10 \\
\hline \hline M5 & $\mathrm{x}$ & $\mathrm{x}$ & $\mathrm{x}$ & $\mathrm{x}$ & & & $\mathrm{x}$ & & & & $\mathrm{x}$ \\
M5 & $\mathrm{x}$ & $\mathrm{x}$ & & & & & $\mathrm{x}$ & & $\mathrm{x}$ & $\mathrm{x}$ & $\mathrm{x}$ \\
M2 & $\mathrm{x}$ & $\mathrm{x}$ & & & & & & $\mathrm{x}$ & & &
\end{tabular}

Having support of this type means that the co-dimension four surface operators on $\mathcal{M}^{4} \times C$, with $\mathcal{M}^{4}$ a fibered product locally of the form $5 \mathbb{R} \times I \times S_{1}^{1} \times S_{2}^{1}$ will be represented as

- surface operators on $\mathcal{M}^{4}$ supported on $\mathcal{M}^{2} \simeq \mathbb{R} \times S_{1}^{1}$,

- line operators in $N=4 \mathrm{SYM}$ on $\mathbb{R} \times I \times C$, supported on $\mathbb{R} \times\{\pi\} \times\{P\}$,

- or line operators in complex CS theory on $\mathbb{R} \times C$, supported on $\mathbb{R} \times\{P\}$.

It easily follows from these observations that the presence of such co-dimension four surface operators modifies the boundary conditions in the two-dimensional sigma model describing the reduction to $\mathbb{R} \times I$. We will now briefly describe the modifications that result from the presence of surface operators of co-dimension four in the effective description as two-dimensional CFT.

\subsection{Resulting modification of the (generalised) AGT-correspondence}

It has been argued in [30] that the presence of co-dimension four surface operators in the sixdimensional $(2,0)$ theory is described after compactification on $C$ as a system where a $4 d$ theory of class $\mathcal{S}$ is coupled to certain two-dimensional gauged linear sigma models (GLSM) on a twodimensional surface $\mathcal{M}^{2}$ in $\mathcal{M}^{4}$. The characteristic feature of the relevant GLSM is the infrared description in terms of nonlinear sigma model with Grassmannians targets. In the simplest case, $\mathfrak{g}=\mathfrak{s l}_{2}$, one gets, for example, the GLSM flowing to the $\mathbb{C P}^{1}$ sigma model.

Our main conjecture concerning the realisation of co-dimension four surface operators in the presence of co-dimension two surface operators within CFT is the following:

Addition of a surface operator of co-dimension four at $P \in C$ gets represented by an insertion of the $\widehat{\mathfrak{s l}}_{2, k}$-module $W_{1 / 2 \hbar}$ at $P$.

\footnotetext{
${ }^{5}$ The lower subscripts in the notation $S_{i}^{1}$ refer to the rotation symmetry used to define the Omega-deformation with parameter $\epsilon_{i}$.
} 
To list the available support for this conjecture, let us start by noticing that it is suggested by a slight generalisation of the picture described in [23]. It was observed in this reference that a system composed out of M5-M5'-branes displays an interesting IR duality relating it to a system of M5-M2-branes, with a collection of M2-branes located at various points on $C$. This IR duality follows from a variant of the Hanany-Witten brane creation effect describing a family of IR-equivalent effective theories obtained by separating the M5-M5'-branes present in the configuration above along the $x_{7}$-axis. In order to get an IR-equivalent effective description one needs to introduce additional $M 2$-branes suspended between the branes getting separated, and extended along the $x_{7}$-axis. The end-points of the $M 2$-branes define a divisor $\sum_{k} P_{k}$ on $C$. It was argued in [23] that this IR duality is expressed in relations between supersymmetric partition functions which get by generalisations of the AGT-correspondence translated into known relations between the conformal blocks of the affine algebra $\widehat{\mathfrak{s l}}_{2, k}$ and $\mathfrak{V i r}_{c}$. The relevant conformal blocks of $\mathfrak{V i r}_{c}$ are defined using insertions of additional degenerate representations $V_{-1 / 2 b}$ with $b^{2}=\hbar$ at the points $P_{1}, \ldots, P_{h}, h=4 g-4+n$. The relations between these two types of conformal blocks follow from a generalisation of Sklyanin's separation of variables (SOV) method.

A simple variant of the set-up considered in [23] is to modify the initial system of M5-M5'branes by having an additional "primordial" M2-brane localised at a point $P_{0} \in C$ in the background, and extended along the $x_{7}$-axis. In the IR dual description one would then get an additional degenerate representation $V_{-1 / 2 b}$ inserted at $P_{0}$ on top of the insertions at $P_{1}, \ldots, P_{h}$. As explained in [7], this is nothing but the representation for conformal blocks of the affine algebra $\widehat{\mathfrak{s l}}_{2, k}$ obtained by the SOV when there is an additional insertion of the $\widehat{\mathfrak{s l}}_{2, k}$-module $W_{1 / 2 \hbar}$ at $P$.

\subsection{Further support}

An alternative argument can be obtained by a suitable generalisation of the arguments leading [30] to describe the effect of additional M2-branes by the insertion of degenerate fields. Addition of such a vertex operator modifies the space of instanton partition functions by tensoring it with the two-dimensional vector space of vacua of the $\mathbb{C P}^{1}$ sigma model. Variation of the position of the co-dimension four surface operator generates a monodromy representation on this two-dimensional vector space. Taking the limit $\epsilon_{2} \rightarrow 0$ results in a partial decoupling of the degrees of freedom on the surface operator and in the bulk, leading to a factorisation of the instanton partition functions into a four-dimensional singular, and a two-dimensional regular part. The $\widehat{\mathfrak{s l}}_{2, k}$-module $W_{1 / 2 \hbar}$ is the unique candidate for an insertion in affine Lie algebra conformal blocks that has the properties (i) to generate a two-dimensional monodromy representation, and (ii) insertions of $W_{1 / 2 \hbar}$ factor off the conformal blocks in the critical level limit. In the following 
section we will describe some elements of this line of arguments in more detail.

It would be nice to check our conjecture (5.5) by localisation calculations, generalising the results for codimension 2 surface operators cited above on the one hand, and the known results for codimension 4 surface operators (see [34] and references therein) on the other hand.

In the reduction to $N=4 \mathrm{SYM}$ one would represent the co-dimension four surface operators in terms of the Wilson and 't Hooft line operators in $N=4$ SYM [35]. The relation of these line operators to the Hecke functors of the geometric Langlands correspondence was extensively discussed in [3]. In the reduction scheme studied by Cordova and Jafferis in [20] one will find the Wilson lines of complex Chern-Simons theory, which should give an alternative view on the conjecture formulated in (5.5).

One may note, finally, that the Hecke functors in the work of Beilinson and Drinfeld are described by the critical level limit of insertions of the modules $W_{-\left(k+h^{\vee}\right) \lambda}$ of $\mathfrak{g}_{k}$ into conformal blocks, where $\lambda$ is the weight of a finite-dimensional representation of ${ }^{L} G$, see [32, Section 20.5] for a review. The modules appearing in (5.5) are associated to the case where $\lambda=1 / 2$ corresponding to the fundamental representation of ${ }^{L} G=\operatorname{PSL}(2, \mathbb{C})$.

\section{Recovering the geometric Langlands correspondence}

Our goal is to elaborate on the relations between generalised versions of the AGTcorrespondence in the presence of surface operators to the geometric Langlands correspondence. We will argue that a direct relation emerges in the Nekrasov-Shatshavili limit $\epsilon_{2} \rightarrow 0$. To this aim we will first observe that the asymptotics of the partition functions $\mathcal{Z}_{\sigma}\left(a ; x, \tau ; \epsilon_{1}, \epsilon_{2}\right)$ contains solutions of the Hitchin eigenvalue equations describing the $\mathcal{D}$-module structures in the geometric Langlands correspondence. We will then discuss how the crucial Hecke eigenvalue property of the relevant $\mathcal{D}$-modules can be understood from the point of view of the NekrasovShatshavili limit of class $\mathcal{S}$ theories with surface operators. Possible interpretations of these phenomena in term of the $2 d$ sigma model with target $\mathcal{M}_{H}(G)$ will be discussed in the next section.

\subsection{Nekrasov-Shatashvili limit}

The limit $\epsilon_{2} \rightarrow 0$ is called Nekrasov-Shatashvili limit following [36]. The relation $k=-h^{\vee}-$ $\epsilon_{2} / \epsilon_{1}$ identifies it with the critical level limit in CFT. In CFT is is known [7, 23] that the chiral partition functions of affine Lie algebra conformal blocks behave in the critical level limit as

$$
\mathcal{Z}_{\sigma}\left(a ; x, \tau ; \epsilon_{1}, \epsilon_{2}\right) \sim e^{-\frac{1}{\epsilon_{2}} \mathcal{Y}_{\sigma}\left(a ; \tau ; \epsilon_{1}\right)} \Psi\left(a ; x, \tau ; \epsilon_{1}\right)\left(1+\mathcal{O}\left(\epsilon_{2}\right)\right),
$$


where

- $\mathcal{Y}_{\sigma}\left(a ; \tau ; \epsilon_{1}\right)$ is the generating function for the Lagrangian submanifold $\mathrm{Op}_{\mathfrak{s l}_{2}}\left(C_{\tau}\right)$ of opers, considered in a related context in [5], with $a$ being the complex Fenchel-Nielsen coordinates for $\mathrm{Op}_{\mathfrak{s l}_{2}}\left(C_{\tau}\right)$ defined from the traces of holonomies along a set of curves on $C$ defining the pants decomposition $\sigma$.

- $\Psi\left(a ; x, \tau ; \epsilon_{1}\right)$ is an eigenfunction of the Hitchin Hamiltonians, $D_{r} \Psi=E_{r} \Psi$, with eigenvalues $E_{r}=E_{r}\left(a ; \tau ; \epsilon_{1}\right)$ obtained from $\mathcal{Y}_{\sigma}\left(a ; \tau ; \epsilon_{1}\right)$ as $E_{r}=\partial_{\tau_{r}} \mathcal{Y}_{\sigma}\left(a ; \tau ; \epsilon_{1}\right)$.

The behaviour (6.6) can be seen as a concrete manifestation of the geometric Langlands correspondence: The points in the character variety having complex Fenchel-Nielsen coordinates $\left(a, \partial_{a} \mathcal{Y}_{\sigma}\left(a ; \tau ; \epsilon_{1}\right)\right)$ may be represented as the holonomies of the $\epsilon_{1}$-opers $\left(\mathcal{E}_{o}, \nabla_{\epsilon_{1}, u}^{\prime}\right)=$ $\left(\mathcal{E}_{o},\left(\epsilon_{1} \partial_{z}+\left(\begin{array}{ll}0 & u \\ 1 & 0\end{array}\right)\right) d z\right)$. To an oper with $u=u^{\sharp}+\sum_{r=1}^{3 g-3+n} E_{r} \vartheta_{r}(x)$, where $u^{\sharp}$ defines a fixed reference oper, and $\vartheta_{r}(x)(d z)^{2}, r=1, \ldots, 3 g-3+n$ is a basis for $H^{0}\left(C, K^{2}\right)$, we may associate the system of equations $D_{r} \Psi=E_{r} \Psi, r=1, \ldots, 3 g-3+n$, defining the $\mathcal{D}$-module associated to the oper $\left(\mathcal{E}_{o}, \nabla_{\epsilon_{1}, u}^{\prime}\right)$ by the geometric Langlands correspondence.

A behaviour of the type (6.6) is easily understood from the point of view of class $\mathcal{S}$-theories with surface operators. In the limit $\epsilon_{2} \rightarrow 0$ one would expect that a modification of the boundary conditions for the gauge fields along a submanifold $\mathcal{M}^{2}$ that stays effectively compact when $\epsilon_{2} \rightarrow 0$ can not affect the leading singular behaviour in this limit.

\subsection{Hecke eigenvalue property in CFT}

In the presence of both types of surface operators one may define further generalisations of the partition functions $\mathcal{Z}_{v}\left(a ; x, \tau ; t ; \epsilon_{1}, \epsilon_{2}\right)$ depending in addition to all the variables considered previously on a variable $t$ which may be identified with the coordinate of a point on $C$, the end-point of an "primordial" M2-brane. We have argued above that such partition functions get represented in CFT as chiral partition functions of conformal blocks with an additional insertion of a degenerate field at $t$. The extra label $v$ refers to the elements of a basis for the space of vacua of the GLSM providing a $4 d+2 d$ Lagrangian description. Considering a fixed choice of pants decomposition we will temporarily suppress the label $\sigma$.

From CFT [7, 23] we know that the partition functions $\mathcal{Z}_{v}\left(a ; x, \tau ; t ; \epsilon_{1}, \epsilon_{2}\right)$ behave in the limit $\epsilon_{2} \rightarrow 0$ as

$$
\mathcal{Z}\left(a ; x, \tau ; t ; \epsilon_{1}, \epsilon_{2}\right) \sim e^{-\frac{1}{\epsilon_{2}} \mathcal{Y}\left(a ; \tau ; \epsilon_{1}\right)} \Psi\left(x ; a, \tau ; \epsilon_{1}\right) \psi_{v}\left(t ; a, \tau ; \epsilon_{1}\right)\left(1+\mathcal{O}\left(\epsilon_{2}\right)\right),
$$

where $\mathcal{Y}\left(a ; \tau ; \epsilon_{1}\right)$ and $\Psi\left(x ; a, \tau ; \epsilon_{1}\right)$ coincide with the functions appearing in 6.6 , and $\psi_{v}(t)=$ $\psi_{v}\left(t ; a, \tau ; \epsilon_{1}\right)$ generate a basis for the space of solutions of the differential equation $\left(\epsilon_{1}^{2} \partial_{t}^{2}-\right.$ 
$u(t)) \psi_{v}(t)=0$ where $u=u(t)$ represents the oper connection as $\epsilon_{1} \partial_{t}+\left(\begin{array}{ll}0 & u \\ 1 & 0\end{array}\right)$. The index $v=+,-$ labels a basis for the space of solutions.

The fact that $\psi_{v}(t)$ factors off is related to the Hecke eigenvalue property, as has been pointed out in [7]. As before we may interpret $\mathcal{Z}_{v}^{*}$ as overlap $\left\langle\delta_{x}^{(2)}, \xi_{a}\right\rangle$, where $\xi_{a} \in \mathcal{H}_{a}^{(2)}$. If (6.7) holds for all $x$, it implies a similar factorisation for all $\xi_{a} \in \mathcal{H}_{a}^{(2)}$. The factor $\psi_{v}(t)$ does furthermore not depend on the choice of vector $\xi_{a} \in \mathcal{H}_{a}^{(2)}$ used to represent the partition function as $\left\langle\delta_{x}^{(2)}, \xi_{a}\right\rangle$. The monodromy generated by analytic continuation of $\psi_{v}(t)$ defines an oper local system $\chi$. Being independent of $\xi_{a} \in \mathcal{H}_{a}^{(2)}$, one may attribute this modification to a modification of the space $\mathcal{H}_{a}^{(2)}$ itself, satisfying the Hecke eigenvalue property.

\subsection{Hecke eigenvalue property from the $t t^{*}$-equations}

Our goal in this section will be to elaborate on the $(4 d+2 d)$-description of the Hecke functors. Due to the fact that the $\Omega$-deformation localises fluctuations to the fixed points of the circle actions, one may think of the limit $\epsilon_{2} \rightarrow 0$ as a decompactification of the hemisphere $B_{\epsilon_{1} \epsilon_{2}}^{4}$ into $B_{\epsilon_{1}}^{2} \times \mathbb{R}^{2}$, where $B_{\epsilon_{1}}^{2}$ is a two-dimensional hemisphere. The surface operators of interest will be supported on $B_{\epsilon_{1}}^{2}$.

The goal is to offer an interpretation for the behaviour (6.7) in four-dimensional terms. Recall that the partition function $\mathcal{Z}_{v}\left(a ; x, \tau, t ; \epsilon_{1}, \epsilon_{2}\right)$ can be interpreted as a partition function of the gauge theory on a $4 \mathrm{~d}$ hemisphere $B_{\epsilon_{1} \epsilon_{2}}^{4}$ with $\Omega$-deformation, defined using boundary conditions

projecting the state created by the gauge theory path integral on $B_{\epsilon_{1} \epsilon_{2}}^{4}$ to an eigenstate of the Wilson-loops [4, 37]. The variables a parameterise the Wilson loop eigenvalues, and the collection of UV gauge couplings of the 4d SUSY gauge theory is denoted by $\tau$. The variables $x$ parameterise the boundary conditions defining a codimension-2 surface operator [23], while the variable $t$ represents the FI parameter $t$ of the GLSM representing the additional surface operator of co-dimension four [30].

\subsubsection{Factorisation}

The degrees of freedom on the surface operator can not contribute to the divergence of the free energy when $\epsilon_{2} \rightarrow 0$ as the two-ellipsoid appearing in this limit ends up having finite volume. We therefore expect that $\mathcal{F}=\log \mathcal{Z}$ behaves as

$$
\mathcal{F} \sim-\frac{1}{\epsilon_{2}} \mathcal{Y}^{4 \mathrm{~d}}\left(a ; \tau ; \epsilon_{1}\right)+\mathcal{W}^{2 \mathrm{~d}}\left(a, \tau ; x, t ; \epsilon_{1}\right)+\ldots,
$$

where $\mathcal{W}^{4 \mathrm{~d}}\left(a ; \tau ; \epsilon_{1}\right)$ is independent of the $2 \mathrm{~d}$ couplings $x$ and $t$. Comparison with (6.6) gives

$$
\mathcal{W}^{2 \mathrm{~d}}\left(a, \tau ; x, t ; \epsilon_{1}\right)=\mathcal{W}^{\mathrm{M} 2}\left(a, \tau ; t ; \epsilon_{1}\right)+\mathcal{W}^{\mathrm{M} 5^{\prime}}\left(a, \tau ; x ; \epsilon_{1}\right),
$$


where $\mathcal{W}^{\mathrm{M} 2}\left(a, \tau ; t ; \epsilon_{1}\right)=\log \psi_{v}\left(t ; a, \tau ; \epsilon_{1}\right)$ and $\mathcal{W}^{\mathrm{M} 5^{\prime}}\left(a, \tau ; x ; \epsilon_{1}\right)=\log \Psi\left(x ; a, \tau ; \epsilon_{1}\right)$. This decoupling can probably be understood as a consequence of the fact that the boundary condition defining a codimension 2 surface operator only involves the bulk fields and not the degrees of freedom used to describe the codimension 4 surface operator.

It is then natural to interpret $\mathcal{Z}_{v}^{2 \mathrm{~d}}\left(a, \tau ; t ; \epsilon_{1}\right):=\psi_{v}(t)$ as the partition function of the GLSM on the lower $2 \mathrm{~d}$ hemisphere $B_{\epsilon_{1}}^{2}$ with $\Omega$-deformation, coupled to the class $\mathcal{S}$ theory in the bulk, and subject to boundary conditions denoted as $\mathfrak{b}_{v}$. Examples of such partition functions have recently been studied in [38], where a close relation was found to the hemisphere partition functions studied in [39, 40]. It was observed that the dependence of $\mathcal{Z}_{v}^{2 \mathrm{~d}}\left(a, \tau ; t ; \epsilon_{1}\right)$ on the FI parameter $t$ is holomorphic. The partition function is furthermore related to an overlap in the topological twisted version of the GLSM as [39, 40]

$$
\mathcal{Z}_{v}^{2 \mathrm{~d}}\left(a, \tau ; t ; \epsilon_{1}\right)=\left\langle\mathfrak{b}_{v} \mid 0\right\rangle
$$

where $|0\rangle$ is the state created by the path integral on the hemisphere with no operator insertion, and $\left\langle\mathfrak{b}_{v}\right|$ is the boundary state associated to the boundary condition $\mathfrak{b}_{v}$.

\subsection{2 $t t^{*}$-equations}

The overlap $\left\langle\mathfrak{b}_{v} \mid 0\right\rangle$ has a natural generalisation obtained by replacing $|0\rangle$ by an other supersymmetric ground state $|1\rangle$ created from $|0\rangle$ by means of the chiral ring generator $v$, $|1\rangle=v|0\rangle$. It has been observed in [41] that $\Pi_{i}^{v}:=\left\langle\mathfrak{b}_{v} \mid i\right\rangle$ represents a horizontal section of the $t t^{*}$-connection of Cecotti and Vafa [42]. In our case we have a single coupling $t$ and a two-dimensional space of SUSY vacua. The $t t^{*}$-connection therefore has the form of a flat $S L(2, \mathbb{C})$-connection $\nabla^{\prime}=\left(\partial_{t}+\mathcal{A}_{t}\right) d t, \nabla^{\prime \prime}=\left(\bar{\partial}_{\bar{t}}+\mathcal{A}_{\bar{t}}\right) d \bar{t}$ [43]. It follows from the holomorphicity observed in [38, 39, 40] that $\mathcal{A}_{\bar{t}}$ must be nilpotent in the basis $\Pi_{i}^{v}$ for the case at hand: This is necessary for the existence of a gauge transformation preserving $\Pi_{0}^{v}$ and relating the connection $\mathcal{A}$ to an oper. The horizontality condition $\nabla^{\prime} \Pi^{v}=0=\nabla^{\prime \prime} \Pi^{v}$ then implies a second order differential equation for $\Pi_{0}^{v}$. The observations above give a physical realisation for the description of opers given in [44].

It follows from the arguments presented in [30] that the couplings $t$ can be identified with local coordinates on the Riemann surface $C_{\tau}$. On physical grounds one would expect that the ordinary differential equation following from the horizontality condition should be non-singular on $C_{\tau}$. This would imply that the $t t^{*}$-connection must be gauge equivalent to an oper connection. The results of [38, 39, 40] provide support for this claim. 


\subsubsection{Fixing the monodromies}

A weakly coupled Lagrangian description of the $4 \mathrm{~d}$ theories of class $\mathcal{S}$ exists when the complex structure of $C_{\tau}$ is near a boundary of the moduli space of Riemann surfaces where $C_{\tau}$ can be represented as a collection of pairs of pants connected by long tubes $T_{1}, \ldots, T_{3 g-3+n}$. The coupled 4d-2d-system will be weakly coupled if $t$ is located on a particular tube $T_{r}$. The Lagrangian description involves a coupling of the GLSM to the $4 \mathrm{~d}$ bulk theory of the form $t a_{r} / \epsilon_{1}$, where $a_{r}$ is the restriction of the scalar field in the vector multiplet associated to $T_{r}$ to the support of the codimension-4 surface operator [30]. The monodromy of the partition function $\mathcal{Z}_{v}^{2 \mathrm{~d}}\left(a ; \tau ; t ; \epsilon_{1}\right)$ corresponding to $t \rightarrow t+2 \pi$ is therefore diagonal, and can be represented by multiplication with $e^{2 \pi v a_{r} / \epsilon_{1}}$. This fixes half of the monodromy of the $t t^{*}$-connection which is enough to fix it completely, being gauge equivalent to an oper connection.

The $t t^{*}$-connection thereby gets identified with the oper local system $\rho$ appearing on one side of the geometric Langlands correspondence. Taking the quotient of the algebra of global differential operators on $\operatorname{Bun}_{G}$ by the ideal generated from $D_{r}-E_{r}, r=1, \ldots, 3 g-3+n$ defines the corresponding D-module $\Delta_{\rho}$ on the other side of the Langlands correspondence [6]. The factorisation (6.7) can be seen as a manifestation of the Hecke eigenvalue property on the level of the solutions of differential equations associated to the D-module $\Delta_{\rho}$.

\section{Sigma model interpretation?}

The discussion in the previous section has revealed close connections between generalisations of the AGT-correspondence in the presence of surface operators and the Beilinson-Drinfeld approach to the geometric Langlands correspondence. We had identified the geometric Langlands correspondence as the limit $\epsilon_{2} \rightarrow 0$ of a description for the spaces of conformal blocks in terms of the quantised character varieties. In this final section we are going to return to the question how the emerging picture is related to the Kapustin-Witten approach to the geometric Langlands correspondence. In Section 7.1 we will raise the question how to describe the limit $\epsilon_{2} \rightarrow 0$ in terms of the $2 d$ sigma model. Sections 7.2 and 7.3 outline a speculative answer to this question. In the rest of this Section we will propose a more explicit comparison between the approaches of Beilinson-Drinfeld and Kapustin-Witten based on similar arguments.

\subsection{Comparison I: Relations between AGT- and geometric Langlands correspondences}

We had argued that the $\mathcal{D}$-modules appearing in this context can be characterised in terms of the spaces of solutions to the corresponding differential equations, physically realised as 
partition functions. In the context of the AGT-correspondence with surface operators we had argued that the spaces $\mathcal{H}_{x}^{(2)}:=\operatorname{Hom}_{\mathcal{M}_{H}(G)}\left(\mathrm{B}_{c c}, \mathrm{~L}_{x}^{(2)}\right)$ can be identified with the fibers of affine algebra conformal blocks over the bundles $\mathcal{E}_{x}$. In CFT we can view the chiral partition functions $\mathcal{Z}(a ; x, \tau ; k)$ solving the $\mathrm{KZB}$ equations as representatives of elements $\varphi_{a}$ of subspaces $\mathcal{C B}_{a}^{\sigma}\left(C, \hat{\mathfrak{g}}_{k}, \mathcal{E}_{x}\right)$ of the spaces $\mathcal{C B}\left(C, \hat{\mathfrak{g}}_{k}, \mathcal{E}_{x}\right)$ conformal blocks having fixed eigenvalues parameterised by $a$ under a maximal commuting subset of the Verlinde line operators.

Modular invariance of the open sigma model TQFT suggests that we can identify $\mathcal{C B}_{a}^{\sigma}\left(C, \hat{\mathfrak{g}}_{k}\right) \simeq$ $\operatorname{Hom}_{\mathcal{M}_{H}(G)}\left(\mathrm{B}_{\mathrm{cc}}, \mathrm{L}_{\sigma, a}^{(1)}\right)$. This identification can be supported by observing that the boundary condition $\mathrm{L}_{\sigma, a}^{(1)}$ fixes the values of some zero modes in the sigma model on the strip, which should lead to an eigenvalue property w.r.t. to the subalgebra of $\mathcal{A}_{B}^{\hbar}$ generated by the corresponding quantised trace functions, associated to the curves defining the pants decomposition $\sigma$.

In the last section we have discussed how the subspaces $\mathcal{C B}_{a}^{\sigma}\left(C, \hat{\mathfrak{g}}_{k}, \mathcal{E}_{x}\right)$ get related to the Heckeeigen $\mathcal{D}$-modules appearing the the geometric Langlands correspondence in the limit $\epsilon_{2} \rightarrow 0$, with local systems representing the "eigenvalue" being represented by families of opers parameterised by the variables $a$. What is not clear, however, is how our observations concerning the appearance of the Hecke eigenvalue property in the limit $\epsilon_{2} \rightarrow 0$ can be understood from the perspective of the sigma model. There appears to be an immediate obstacle: The sigma model considered in [3] in the context of the geometric Langlands correspondence are A-models with symplectic structure $\omega_{K}$, and the cc-brane has Chan-Paton curvature $\omega_{J}$. A different choice appears in the Nekrasov-Witten approach to the AGT-correspondence, where the A-model with symplectic structure $\frac{\epsilon_{2}}{\epsilon_{1}} \omega_{I}$ is used. There is no obvious parameter allowing us to move continuously between these two cases.

In view of the fact that within CFT one can obtain the geometric Langlands correspondence in the critical level limit it seems very natural to ask if this can be understood within the $2 d$ sigma model with target $\mathcal{M}_{H}(G)$. Starting from Subsection 7.2 we'll speculate about a possible way to see this.

\subsection{Hyperkähler rotation}

It is tempting to modify the 2d TQFT set-up of Nekrasov and Witten using the hyperkähler ${ }^{(g)}$ rotations coming from the circle action $\varphi \rightarrow e^{i \theta} \varphi$ on Hitchin's moduli spaces. It is shown in [45. Section 9] that this circle action can be complexified into a $\mathbb{C}^{*}$-action relating all complex and symplectic structures in the family $\left(I_{\zeta}, \Omega_{\zeta}\right)$ apart from $\zeta=0, \infty$. The hyperkähler rotations allow us to identify the key geometric structure on $\mathcal{M}_{H}(G)$ for $\zeta \neq 0, \infty$ in the sense that the rotations can be represented by diffeomorphisms of $\mathcal{M}_{H}(G)$ [45, Proposition (9.1)]. This amounts to reparametrizations of the sigma model fields, which may not affect the physical 
content.

Invariance of the sigma model path integral under field redefinitions may be a subtle issue. The circle action $\varphi \rightarrow e^{i \theta} \varphi$ should be easy to understand, but it is not clear to us if the complexification to a $\mathbb{C}^{*}$-action is easy to understand as a symmetry of the sigma model TQFT in a suitable sense. What we'd like to point out is that validity of a certain form of invariance of the topological sigma models with target $\mathcal{M}_{H}(G)$ under the $\mathbb{C}^{*}$-action would give us a simple way to interpolate between the sigma models considered in [3] and [4], respectively. In this way one could get an attractive explanation of the observation made in the previous section.

We are interested in open A-models on surfaces with boundaries with boundary conditions being either of the canonical coisotropic or Lagrangian type. We may use the family of holomorphic symplectic forms $\Omega_{\zeta}$ to define a family of A-models with symplectic structure $\operatorname{Im}\left(\Omega_{\zeta}\right)$ having coisotropic branes with Chan-Paton curvature $\operatorname{Re}\left(\Omega_{\zeta}\right)$. Submanifolds of $\mathcal{M}_{H}(G)$ which are Lagrangian with respect to $\Omega_{\zeta}$ define branes in this family of A-models. If an appropriate form of $\mathbb{C}^{*}$-invariance of the sigma model TQFT holds, one would expect that the partition functions $\mathcal{Z}_{\zeta}$ defined in this family of A-models are in fact $\zeta$-independent.

One should note, however, that all Lagrangian submanifolds we use to define boundary conditions will have to be varied consistently to keep $\mathcal{Z}_{\zeta}$ unchanged. The submanifolds of our interest are the orbits of the complex Fenchel-Nielsen ${ }^{(g)}$ twist flows in the character variety, considered as submanifolds of $\mathcal{M}_{H}(G)$ via NAH-correspondence and holonomy map. Considering a fixed orbit in the character variety, one gets a one-parameter family of submanifolds of $\mathcal{M}_{H}(G)$ upon varying the hyperkähler parameter $\zeta$.

Given we have invariance under the $\mathbb{C}^{*}$-action, we could combine variations of the coupling parameter $\epsilon_{1} / \epsilon_{2}$ with a suitable hyperkähler rotation in such a way that we obtain a one-parameter family of topological A-models with target $\mathcal{M}_{H}(G)$ that interpolates between the ones considered in [4] and [3], respectively. This could be done by setting $\zeta=\epsilon_{2} \xi$. The expression for $\hat{\omega}_{\zeta}=\frac{\epsilon_{2}}{\epsilon_{1}} \operatorname{Im}\left(\Omega_{\zeta}\right)$ reduces in the limit $\epsilon_{2} \rightarrow 0$ to $\hat{\omega}_{\zeta}=\frac{1}{2 \epsilon_{1}|\xi|^{2}}\left(\operatorname{Re}(\zeta) \omega_{K}-\operatorname{Im}(\zeta) \omega_{J}\right)$, reproducing for real $\xi$ the symplectic form used to define the A-model studied in [3].

\subsection{Nekrasov-Shatashvili limit of the branes $\mathrm{L}_{\sigma, a}^{(1)}$}

In order to see that the resulting scenario may indeed resolve the puzzle stated above, let us first note that the (A,B,A) branes $\mathrm{L}_{\sigma, a}^{(1)}$ considered in Section (4.3) can easily be generalised into $(B, A, A)_{\zeta}$-branes $\mathrm{L}_{\sigma, a}^{(1) \zeta}$ by using the $\zeta$-dependent $\mathrm{NAH}$-correspondence in their definition. Choosing $\zeta$ in an $\hbar$-dependent way, as suggested above, would turn them into branes $\mathrm{L}_{\sigma, a}^{(1) \hbar}$ in the family of A-models with hyperkähler parameter $\hbar=\epsilon_{1} / \epsilon_{2}$. 
We will observe that something interesting happens in the Nekrasov-Shatashvili of the family of branes $\mathrm{L}_{\sigma, a}^{(1) \hbar}$ : The branes $\mathrm{L}_{\sigma, a}^{(1) \hbar}$ will have a well-defined limit $\hbar \rightarrow \infty$ if the parameters $a$ are scaled in this limit as $a_{r}=\frac{\epsilon_{1}}{\epsilon_{2}} \check{a}_{r}$ with $\check{a}_{r}$ finite. In order to understand the limit $\epsilon_{2} \rightarrow 0$ one mainly needs to study the WKB approximation for the holonomy of $\partial_{u}+\mathcal{A}_{u}$ where $\mathcal{A}_{u}=A_{u}+\frac{1}{\epsilon_{2}} \varphi$. By gauge transformations one can always locally reach the form $\mathcal{A}_{u}=\left(\begin{array}{cc}0 & u_{\epsilon_{2}} \\ 1 & 0\end{array}\right)$. The function $u_{\epsilon_{2}}(t)$ appearing in the upper right matrix element of $\mathcal{A}_{u}$ will in general only be meromorphic in $u$, but the residues are of order $\epsilon_{2}$. We note that $A_{u}$ contributes only in subleading orders of the expansion. It follows easily from these facts that $\vartheta_{\epsilon_{2}}(t)=\frac{1}{2} \operatorname{tr}\left(\varphi^{2}\right)+\mathcal{O}\left(\epsilon_{2}\right)$. To leading order in $\epsilon_{2}$ one may therefore represent the solutions $s$ to $\left(\partial_{u}+\mathcal{A}_{u}\right) s_{\epsilon_{2}}=0$ in the form

$$
s_{\epsilon_{2}}(t) \sim e^{ \pm \frac{1}{\epsilon_{2}} \int_{\mathcal{C}_{t}} \lambda}
$$

where $\mathcal{C}_{t}$ is a path on the spectral curve $\Sigma$ ending at a lift $\hat{t} \in \Sigma$ of $t \in \mathbb{C}$, and $\lambda$ is the canonical differential on $\Sigma$. The conclusion is that the rescaled Fenchel-Nielsen length coordinates $\check{a}_{r}$ behave in the limit $\epsilon_{2} \rightarrow 0$ as $\check{a}_{r}=\frac{1}{\epsilon_{1}} \mathrm{a}_{r}+\mathcal{O}\left(\epsilon_{2}\right)$, where $\mathrm{a}_{r}$ are the periods of the differential $\lambda$ defined along a Lagrangian subspace of a canonical homology basis determined by $\sigma$.

One may in this sense view the "quantum periods" $\check{a}_{r}$ as deformations of the angle variables $\mathrm{a}_{r}$ for the Hitchin integrable system. It is interesting to note that the dependence on $\sigma$ disappears in the limit $\epsilon \rightarrow 0$ : Even if the definition of the coordinates $a_{r}$ carries a residual dependence on $\sigma$, this is not the case for the fiber of Hitchin's fibration determined by the values of the $a_{r}$. In this way we are led to the conclusion that the scaling limits of the branes $\mathrm{L}_{\sigma, a}^{(1) \hbar}$ get represented by the (B,A,A)-branes supported on the fibers $F_{\left(u_{a}, 0\right)}$ of Hitchin's (first) fibration with trivial Chan-Paton bundle over a point $u_{\mathrm{a}}$ on the Hitchin base $\mathcal{B}$ determined by the coordinates $\mathrm{a}_{r}$.

Let us finally note that one could discuss the branes $L_{x}^{(2) \hbar}$ in a similar way. It is easy to see that one obtains the branes $L_{x}^{(2)}$ supported on fibers of Hitchin's second fibration when $\hbar \rightarrow 0$.

\subsection{Comparison II: Relation between Kapustin-Witten approach and CFT?}

Any comparison between the approaches of Beilinson-Drinfeld and Kapustin-Witten will need to address the following point. Hecke-eigenbranes are described in [3] as skyscraper sheaves on $\mathcal{M}_{H}(G)$. However, in order to use SYZ mirror symmetry on the Hitchin fibration, Kapustin and Witten use the representation of $\mathcal{M}_{H}(G)$ and $\mathcal{M}_{H}\left({ }^{L} G\right)$ adapted to the complex structure $I$, whereas the Beilinson-Drinfeld approach considers ${ }^{L} G$-local systems $\left(\mathcal{E}, \nabla^{\prime}\right)$ on one side of the correspondence. In order to relate the two, one needs to use the NAH-correspondence. If $\mu$ represents a point of $\mathcal{M}_{H}(G)$, represented as a torus fibration, let $\check{\mathrm{F}}_{\mu}$ be the corresponding skyscraper sheaf in the B-model, and $\mathrm{F}_{\mu}$ be the Hitchin fiber in the SYZ-dual A-model. In order to formulate a conjectural relation between the two approaches we need to use the (inverse of the) NAH-correspondence to find the point $\mu(\chi) \in \mathcal{M}_{H}(G)$ associated to the local system $\chi$. 
A natural guess for a possible relation between the approaches of Beilinson-Drinfeld and Kapustin-Witten could then be the validity of the isomorphism of $\mathcal{D}$-modules

$$
\begin{aligned}
\operatorname{Hom}_{\mathcal{M}_{H}(G)}\left(\mathrm{B}_{\mathrm{cc}}, \mathrm{F}_{\mu(\chi)}\right) & \simeq \mathcal{C B}\left(C, \hat{\mathfrak{g}}_{-h^{\vee}}, \mathcal{E}\right) \text { for } \mathcal{E} \in \operatorname{Bun}_{G}^{\mathrm{vs}} \\
& \simeq\left[\text { Fiber of } \Delta_{\chi} \text { over } \mathcal{E} \in \operatorname{Bun}_{G}^{\mathrm{vs}}\right]
\end{aligned}
$$

where $\operatorname{Bun}_{G}^{\text {vs }}$ is the space of "very stable" bundles $\mathcal{E}$, bundles that do not admit a nilpotent Higgs field, and $\Delta_{\chi}$ is the $\mathcal{D}$-module represented by $\mathcal{C B}\left(C, \hat{\mathfrak{g}}_{-h^{\vee}}, \mathcal{E}\right)$. The right hand side does not depend on the choice of $\mathcal{E}$ due to the existence of a canonical flat connection identifying fibers associated to different $\mathcal{E} \in \operatorname{Bun}_{G}^{\mathrm{vs}}$. The dimension of $\mathcal{C B}\left(C, \hat{\mathfrak{g}}_{-h^{\vee}}, \mathcal{E}\right)$ may jump away from $\operatorname{Bun}_{G}^{\mathrm{vs}}$, see [6, Section 9.5] for a discussion.

At this point we may note another puzzle arising in the comparison of the approaches of Beilinson-Drinfeld and Kapustin-Witten. As noted above, and illustrated by the examples studied in [7], there is a somewhat discontinuous behaviour of the $\mathcal{D}$-modules appearing in the Beilinson-Drinfeld approach to the geometric Langlands correspondence away from the subvariety of opers within $\mathcal{M}_{d R}\left({ }^{L} G\right)$, described by the appearance of a number of additional degenerate representations in the representation of the $\mathcal{D}$-modules as conformal blocks. No such discontinuous behaviour is seen in the approach of Kapustin and Witten.

\subsection{Conformal limit}

We would also like to suggest a way which might lead to an answer for the questions raised in Subsection 7.4 above. It is based on the observation made in [46, 44] that the NAH correspondence may simplify drastically in the conformal limit where the parameter $R$ introduced into the NAH correspondence by scaling $\varphi \rightarrow R \varphi$ is sent to zero together with the hyperkähler parameter $\zeta$ such that $\zeta / R$ stays finite. As a preparation we'd here like to discuss the possible relevance of this limit. The issues are similar, but non-identical to the ones concerning the possible relevance of hyperkähler rotations discussed in Section 7.2. motivating a separate discussion.

Replacing $\varphi \rightarrow R \varphi$ defines a one-parameter family of deformations of the NAH correspondence, leading to an apparent modification of the hyperkähler metric on $\mathcal{M}_{H}(G)$. However, the parameter $R$ is inessential for the geometry of Hitchin's moduli spaces in the sense that hyperkähler metrics associated to different values of $R$ are related by diffeomorphisms. One may define one-parameter families of sigma model actions $S_{R}$ using the hyperkähler metrics on $\mathcal{M}_{H}(G)$ obtained from the modified NAH correspondence. However, being constructed out of hyperkähler metrics related by diffeomorphisms, one may be tempted to identify two actions $S_{R_{1}}$ and $S_{R_{2}}$ differing only in the choice of the parameter $R$ as physically equivalent Lagrangian 
representations for the same sigma model QFT. In suitable coordinates like $u=\frac{1}{2} \operatorname{tr}\left(\varphi^{2}\right)$ one finds that the relevant diffeomorphism is realised as a simple scaling $u \rightarrow u r^{2}$. The corresponding field redefinition should indeed lead to a rescaling of partition functions by inessential overall factors only.

If the partition functions $Z_{R}(u)$ defined using the actions $S_{R}$ depend on a boundary parameter $u$ associated to a coordinate for $\mathcal{M}_{H}(G)$ scaling under $\varphi \rightarrow R \varphi$ as $u \rightarrow u r^{2}$, we find that the $R$-independence of the sigma model metric modulo diffeomorphisms is expressed in the fact that $Z_{r R}(u) \propto Z_{R}\left(u r^{2}\right)$, again possibly up to inessential overall factors.

A partition function $Z^{\prime}(\chi)$ in the boundary B-model with a boundary condition defined by a point $\chi$ on $\mathcal{M}_{d R}\left({ }^{L} G\right)$ may be represented in terms of a partition function $Z_{R}^{\prime \prime}\left(\mu_{R, \zeta}(\chi)\right)$ depending on a point $\mu_{R, \zeta}(\chi) \in \mathcal{M}_{H}\left({ }^{L} G\right)$ since the $R$-dependence resulting from the modification of the NAH correspondence is compensated by the corresponding modification of the sigma model action. This observation may be useful if there is a limit where $\mu_{R, \zeta}(\chi)$ simplifies considerably. We will see that such a limit is the so-called conformal limit $R \rightarrow 0, \zeta \rightarrow 0$ keeping $\zeta / R$ finite. This will lead to an interesting reformulation of the proposed relation (7.11), as we shall now discuss.

\subsection{Relation between Kapustin-Witten approach and CFT, more explicitly}

We will mostly restrict attention to the case where the local systems $\chi$ are opers $\rho_{u}=\left(\mathcal{E}_{o},\left(\partial_{t}+\right.\right.$ $\left.\left(\begin{array}{ll}0 & u \\ 1 & 0\end{array}\right)\right) d t$, playing a basic role in the approach of Beilinson and Drinfeld. It is interesting to note that the conformal limit of the NAH correspondence becomes particularly simple in this case [44], relating opers $\rho_{u}$ to Higgs pairs of a very particular form. For ${ }^{L} G=\operatorname{PSL}(2, \mathbb{C})$ one finds Higgs pairs $(\mathcal{E}, \varphi)$ of the form $\left(K^{1 / 2} \oplus K^{-1 / 2},\left(\begin{array}{ll}0 & u \\ 1 & 0\end{array}\right) d z\right)$, where $u$ is the quadratic differential representing $\rho_{u}$ in the Fuchsian uniformisation of $C$. Passing to the description of $\mathcal{M}_{H}\left({ }^{L} G\right)$ as a torus fibration represented by pairs $\left(\Sigma_{u}, \mathcal{L}\right)$, where $\Sigma_{u}$ is the spectral curve associated to a point $u \in \mathcal{B}$ of the Hitchin base, and $\mathcal{L}$ is a line bundle on $\Sigma_{u}$, one gets the line bundle $\mathcal{L}_{0}=\pi^{*}\left(K^{1 / 2}\right)$, with $\pi: \Sigma_{u} \rightarrow C$ being the covering projection. This line bundle represents a canonical "origin" of the Jacobian/Prym parametrising the choices of $\mathcal{L}$ [47].

Let $\check{\mathrm{F}}_{(u, 0)}$ be the skyscraper sheaf on $\mathcal{M}_{H}\left({ }^{L} G\right)$ supported at the point $\left(\Sigma_{u}, \mathcal{L}_{0}\right)$ and let $\mathrm{F}_{(u, 0)}$ be the fiber of the Hitchin fibration which is SYZ dual to $\check{F}_{(u, 0)}$. The possible relation (7.11) between the approaches of Beilinson-Drinfeld and Kapustin-Witten may then be formulated more explicitly as

$$
\operatorname{Hom}_{\mathcal{M}_{H}(G)}\left(\mathrm{B}_{\mathrm{cc}}, \mathrm{F}_{(u, 0)}\right) \simeq\left[\text { Fiber of } \Delta_{\rho_{u}} \text { over } \mathcal{E} \in \operatorname{Bun}_{G}^{\mathrm{vs}}\right]
$$

We remark that the image of generic points $(u, 0) \in \mathcal{M}_{H}\left({ }^{L} G\right)$ under the NAH correspondence 
will be represented by an oper if and only if the map from $\mathcal{M}_{H}\left({ }^{L} G\right)$ to $\mathcal{M}_{d R}\left({ }^{L} G\right)$ is defined using the conformal limit of the non-abelian Hodge correspondence 6 This, and the relevance of this limit in Section 6.3, indicate that this limit is well-suited for formulating the relation between the approaches of Beilinson-Drinfeld and Kapustin-Witten.

Concerning the generalisation of (7.12) to more general local systems $\chi$ we conjecture that there exist natural stratifications of $\mathcal{M}_{d R}\left({ }^{L} G\right)$ and $\mathcal{M}_{H}\left({ }^{L} G\right)$, having strata related to each other by the conformal limit of the NAH correspondence. This would allow us to extend the relation (7.12) to generic irreducible local systems, linking the discontinuous behaviour of the $\mathcal{D}$-modules appearing in the geometric Langlands correspondence to the passage from one stratum to another. We plan to return to this point in a forthcoming publication.

\subsection{Outlook}

We will elsewhere discuss available evidence for the existence of relations of the form

$$
\mathcal{H}_{x}^{(2)} \simeq \int^{\oplus} d \mu_{\sigma}(a) \mathcal{H}_{\sigma, a}^{(1)},
$$

and for the existence of linear relations between the spaces $\mathcal{H}_{\sigma, a}^{(1)}$ associated to different pants decompositions $\sigma$. This restores a weaker version of $\sigma$-independence within the story associated to nonzero $\epsilon_{2}$.

The geometric Langlands correspondence is sometimes presented as an analog of the spectral decompositions of spaces of automorphic forms appearing in the classical Langlands program. We view the contents of this note as hints that it may not be outrageous to dream of a variant of the geometric Langlands program extending it by transcendental and analytic aspects. The transcendental aspects involve the partition functions representing solutions to the systems of differential equations defined by the $\mathcal{D}$-modules, and the analytic aspects concern spectral decompositions as proposed in (7.13). Identifying the partition functions as analogs of the automorphic forms would strengthen the analogies to the classical Langlands program even further. The partition functions represent the bridge between the algebraic structures of $\mathcal{M}_{H}(G)$ associated to the representation as moduli space $\mathcal{M}_{d R}(G)$ of local systems, and as character variety $\mathcal{M}_{B}(G)$, respectively. In this way one may expect to get a larger picture unifying topological and complex structure dependent aspects of the geometric Langlands program.

We plan to discuss these matters, the interpretation as "quantum geometric Langlands duality",

\footnotetext{
${ }^{6}$ The direction "if" was shown in [44]. The image of points $(u, 0) \in \mathcal{M}_{H}\left({ }^{L} G\right)$ under NAH consists of connections with real holonomy, intersecting the variety of opers only discretely. We thank A. Neitzke for this remark.
} 
and the relation to another incarnation of Langlands duality patterns referred to as modular duality in [7] in forthcoming publications.

Let us finally note that recent progress on the geometric Langlands program from the gauge theory side has been described in [48, 49]. It should be interesting to analyse the relations to our work.

Acknowledgements: A.B would like to thank D. Ben-Zvi, M. Mulase, A. Neitzke and R. Wentworth for discussions and the organizers of String-Math 2016 for putting together a stimulating conference. A.B would also like to thank L'Institut Henri Poincaré (Paris) and ICTS-TIFR (Bangalore) for hospitality during visits when this work was in progress.

J.T. would like to thank the organizers of String-Math 2016 for setting up an inspiring conference and the opportunity to present this work, and M. Mulase, A. Neitzke for discussions. Special thanks to E. Frenkel for various discussions, for communicating the content of his unpublished work [28], and for critical remarks on the draft.

This work was supported by the Deutsche Forschungsgemeinschaft (DFG) through the collaborative Research Centre SFB 676 "Particles, Strings and the Early Universe", project A10.

\section{A. Hitchin's moduli spaces}

We assume that $G=S L(2)$, and that $C$ is a Riemann surface with genus $g$ and $n$ punctures.

Hitchin moduli space $\mathcal{M}_{H}(G)$ [45]. Moduli space of pairs $(\mathcal{E}, \varphi)$, where $\mathcal{E}=\left(E, \bar{\partial}_{\mathcal{E}}\right)$ is a holomorphic structure on a smooth vector bundle $E$, and $\varphi \in H^{0}(C, \operatorname{End}(\mathcal{E}) \otimes K)$. The moduli space of such pairs modulo natural gauge transformations is denoted by $\mathcal{M}_{H}(G)$.

Hitchin's integrable system [45]. Given $(\mathcal{E}, \varphi)$ one constructs the spectral curve $\Sigma=$ $\left\{(u, v) ; v^{2}=\frac{1}{2} \operatorname{tr}\left(\varphi^{2}\right)\right\} \subset T^{*} C$, and the line bundle $\mathcal{L}$ representing the cokernel of $\varphi-v$. One may reconstruct $(\mathcal{E}, \varphi)$ from $(\Sigma, \mathcal{L})$ as $\mathcal{E}=\pi_{*}(\mathcal{L})$ and $\varphi=\pi_{*}(v)$. This describes $\mathcal{M}_{H}(G, C)$ as a torus fibration over the base $\mathcal{B} \simeq H^{0}\left(C, K^{2}\right)$, with fibres representing the choices of $\mathcal{L}$ identified with the Jacobian of $\Sigma$ if $G=G L(2)$, and with the Prym variety if $G=S L(2)$. Natural coordinates for the base $\mathcal{B}$ are provided by Hitchin's Hamiltonians, defined by expanding $\frac{1}{2} \operatorname{tr}\left(\varphi^{2}\right)=\sum_{r=1}^{3 g-3+n} \vartheta_{r} H_{r}$, with $\left\{\vartheta_{r}, r=1, \ldots, 3 g-3+n\right\}$ being a basis for $H^{0}\left(C, K^{2}\right)$.

Local systems. Pairs $\left(\mathcal{E}, \nabla_{\epsilon}^{\prime}\right)$, where $\mathcal{E}$ is a holomorphic vector bundle as above, and $\nabla_{\epsilon}^{\prime}$ is a holomorphic $\epsilon$-connection, satisfying $\nabla_{\epsilon}^{\prime}(f s)=\epsilon(\partial f) s+f \nabla_{\epsilon}^{\prime} s$ for functions $f$ and smooth sections $s$ of $E$. The moduli space of such pairs is denoted $\mathcal{M}_{d R}(G)$. Local systems are here often identified with the corresponding flat bundles, systems of local trivialisations with constant transitions functions, or the representations of the fundamental group (modulo conjugation) 
obtained as holonomy of $\left(\mathcal{F}, \nabla_{\epsilon}^{\prime}\right)$, leading to the isomorphism between $\mathcal{M}_{d R}(G)$ and the

Character variety $\mathcal{M}_{B}(G)$ : The space of representations of $\pi_{1}(C)$ into $G$, modulo overall conjugation, as algebraic variety described as a GIT quotient $\mathbb{C}\left[\operatorname{Hom}\left(\pi_{1}(C), G\right]^{G}\right.$.

Opers. Special local systems, where $\mathcal{E}=\mathcal{E}_{\text {op }}$, the unique extension $0 \rightarrow K^{1 / 2} \rightarrow \mathcal{E}_{\text {op }} \rightarrow$ $K^{-1 / 2} \rightarrow 0$ allowing a holomorphic connection $\nabla_{\epsilon}^{\prime}$ of the form $\nabla_{\epsilon}^{\prime}=d z\left(\epsilon \partial_{z}+\left(\begin{array}{ll}0 & u \\ 1 & 0\end{array}\right)\right)$.

Non-Abelian Hodge (NAH) correspondence [45, 50]. Given a Higgs pair $(\mathcal{E}, \varphi)$, there exists a unique harmonic metric $h$ on $E$ satisfying $F_{\mathcal{E}, h}+R^{2}\left[\varphi, \varphi^{\dagger h}\right]=0$ where $F_{\mathcal{E}, h}$ is the curvature of the unique $h$-unitary connection $D_{\mathcal{E}, h}$ having $(0,1)$-part $\bar{\partial}_{\mathcal{E}}$. One may then form the corresponding two-parameter family of flat connections $\nabla_{\zeta, R}=\zeta^{-1} R \varphi+D_{\mathcal{E}, h}+R \zeta \varphi^{\text {th }}$. Decomposing $\nabla_{\zeta, R}$ into the $(1,0)$ and $(0,1)$-parts defines a pair $\left(\mathcal{F}, \nabla_{\epsilon}^{\prime}\right)$ consisting of $\mathcal{F}=\left(E, \bar{\partial}_{\mathcal{F}}\right)$ and the $\epsilon$-connection $\nabla_{\epsilon}^{\prime}=\epsilon \nabla^{\prime}=\epsilon \partial_{\mathcal{E}, h}+\varphi$, with $\epsilon=\zeta / R$, holomorphic in the complex structure defined by $\bar{\partial}_{\mathcal{F}}$.

Hyperkähler structure [45]. There exists a $\mathbb{P}^{1}$ worth of complex structures $I_{\zeta}$ and holomorphic symplectic structures $\Omega_{\zeta}$. The latter are defined as $\Omega_{\zeta}=\frac{1}{2} \int_{C} \operatorname{tr}\left(\delta \mathcal{A}_{\zeta} \wedge \delta \mathcal{A}_{\zeta}\right)$. A triplet of symplectic forms $\left(\omega_{I}, \omega_{J}, \omega_{K}\right)$ can be defined by expanding $\Omega_{\zeta}$ as $\Omega_{\zeta}=\frac{1}{2 \zeta}\left(\omega_{J}+i \omega_{K}\right)+i \omega_{I}+$ $\frac{1}{2} \zeta\left(\omega_{J}-i \omega_{K}\right)$. The corresponding complex structures are $I_{\zeta}=\frac{1}{1+|\zeta|^{2}}\left(\left(1-|\zeta|^{2}\right) I-i(\zeta-\bar{\zeta}) J-\right.$ $(\zeta+\bar{\zeta}) K)$.

Complex Fenchel-Nielsen coordinates [5]. Darboux coordinates for $\mathcal{M}_{B}(G)$ associated to pants decompositions $\sigma$ of $C$ obtained by cutting along closed curves $\gamma_{i}, i=1, \ldots, 3 g-$ $3+n$. The complex length coordinates parameterise the trace functions $L_{i}=\operatorname{tr}\left(\rho\left(\gamma_{i}\right)\right)$ as $L_{r}=2 \cosh \left(a_{r} / 2\right)$. One may define canonically conjugate coordinates $\kappa_{r}$ such that the natural Poisson structure gets represented as $\left\{a_{r}, \kappa_{s}\right\}=\delta_{r, s},\left\{a_{r}, a_{s}\right\}=0=\left\{\kappa_{r}, \kappa_{s}\right\}$.

\section{References}

[1] L. F. Alday, D. Gaiotto, and Y. Tachikawa, Liouville Correlation Functions from Four-dimensional Gauge Theories, Lett.Math.Phys. 91 (2010) 167-197, [arXiv:0906.3219].

[2] N. Nekrasov, BPS/CFT correspondence: non-perturbative Dyson-Schwinger equations and qq-characters, JHEP 03 (2016) 181, arXiv: 1512.0538].

[3] A. Kapustin and E. Witten, Electric-Magnetic Duality And The Geometric Langlands Program, Commun. Num. Theor. Phys. 1 (2007) 1-236, [hep-th/0604151]. 
[4] N. Nekrasov and E. Witten, The Omega Deformation, Branes, Integrability, and Liouville Theory, JHEP 1009 (2010) 092, [arXiv: 1002.0888].

[5] N. Nekrasov, A. Rosly, and S. Shatashvili, Darboux coordinates, Yang-Yang functional, and gauge theory, Nucl. Phys. Proc. Suppl. 216 (2011) 69-93, [arXiv:1103.3919].

[6] E. Frenkel, Lectures on the Langlands Program and Conformal Field Theory, in Frontiers in Number Theory, Physics, and Geometry II (P. Cartier, P. Moussa, B. Julia, and P. Vanhove, eds.), pp. 387-533. Springer Berlin Heidelberg, Berlin, Heidelberg, 2007.

[7] J. Teschner, Quantization of the Hitchin moduli spaces, Liouville theory, and the geometric Langlands correspondence I, Adv. Theor. Math. Phys. 15 (2011) 471-564, [arXiv:1005.2846].

[8] D. Gaiotto and E. Witten, Knot Invariants from Four-Dimensional Gauge Theory, Adv. Theor. Math. Phys. 16 (2012), no. 3, 935-1086, [arXiv:1106.4789].

[9] T. Kimura and V. Pestun, Quiver W-algebras, arXiv:1512.0853.

[10] M. Aganagic, E. Frenkel, and A. Okounkov, Quantum q-Langlands Correspondence, arXiv:1701.0314,

[11] D. Arinkin and D. Gaitsgory, Singular support of coherent sheaves and the geometric Langlands conjecture, Selecta Mathematica 21 (2015), no. 1, 1-199.

[12] B. Feigin and E. Frenkel, Affine Kac-Moody algebras at the critical level and Gelfand-Dikii algebras, Int. J. Mod. Phys. A7, Suppl. 1A (1992) 197-215.

[13] D. Arinkin, Irreducible connections admit generic oper structures, ArXiv e-prints (Feb., 2016) [arXiv:1602.0898].

[14] D. Gaitsgory, Outline of the proof of the geometric Langlands conjecture for $G L_{2}$, Astérisque (2015), no. 370, 1-112.

[15] E. Witten, Geometric Langlands From Six Dimensions, arXiv:0905.2720.

[16] D. Gaiotto, G. W. Moore, and A. Neitzke, Wall-crossing, Hitchin systems, and the WKB approximation, Adv. Math. 234 (2013) 239-403.

[17] D. Gaiotto and E. Witten, Supersymmetric Boundary Conditions in N=4 Super Yang-Mills Theory, J. Statist. Phys. 135 (2009) 789-855, [arXiv:0804.2902]. 
[18] D. Gaiotto and E. Witten, S-Duality of Boundary Conditions In N=4 Super Yang-Mills Theory, Adv. Theor. Math. Phys. 13 (2009), no. 3, 721-896, [arXiv:0807.3720].

[19] D. Ben-Zvi and D. Nadler, Betti Geometric Langlands, ArXiv e-prints (June, 2016) arXiv:1606.0852].

[20] C. Cordova and D. L. Jafferis, Toda Theory From Six Dimensions, arXiv: 1605.0399.

[21] V. Pestun, Localization of gauge theory on a four-sphere and supersymmetric Wilson loops, Commun. Math. Phys. 313 (2012) 71-129, [arXiv:0712.2824].

[22] L. F. Alday and Y. Tachikawa, Affine SL(2) conformal blocks from 4 d gauge theories, Lett.Math.Phys. 94 (2010) 87-114, [arXiv:1005.4469].

[23] E. Frenkel, S. Gukov, and J. Teschner, Surface Operators and Separation of Variables, JHEP 01 (2016) 179, arXiv:1506.0750].

[24] A. Braverman, Instanton counting via affine Lie algebras. 1. Equivariant J functions of (affine) flag manifolds and Whittaker vectors, in Algebraic Structures and Moduli Spaces, vol. 38 of CRM Proc. Lecture Notes, pp. 113-132, Amer. Math. Soc., Providence, 2004.

[25] C. Kozcaz, S. Pasquetti, F. Passerini, and N. Wyllard, Affine sl(N) conformal blocks from $N=2 S U(N)$ gauge theories, JHEP 1101 (2011) 045, [arXiv:1008.1412].

[26] A. Negut, Affine Laumon Spaces and the Calogero-Moser Integrable System, arXiv:1112.1756.

[27] S. Nawata, Givental J-functions, Quantum integrable systems, AGT relation with surface operator, Adv. Theor. Math. Phys. 19 (2015) 1277-1338, [arXiv:1408.4132].

[28] E. Frenkel, On the brane interpretation of conformal blocks, available at http://edwardfrenkel.com/confblocks.pdf (2010).

[29] A. Kapustin, A Note on Quantum Geometric Langlands Duality, Gauge Theory, and Quantization of the Moduli Space of Flat Connections, arXiv:0811.3264.

[30] L. F. Alday, D. Gaiotto, S. Gukov, Y. Tachikawa, and H. Verlinde, Loop and surface operators in N=2 gauge theory and Liouville modular geometry, JHEP 1001 (2010) 113, [arXiv:0909.0945].

[31] N. Drukker, J. Gomis, T. Okuda, and J. Teschner, Gauge Theory Loop Operators and Liouville Theory, JHEP 1002 (2010) 057, [arXiv:0909.1105]. 
[32] E. Frenkel and D. Ben-Zvi, Vertex algebras and algebraic curves. Providence, USA: Am. Math. Soc. (2004) 400 p, 2004.

[33] N. Drukker, D. R. Morrison, and T. Okuda, Loop operators and S-duality from curves on Riemann surfaces, JHEP 09 (2009) 031, arXiv:0907.2593].

[34] J. Gomis, B. Le Floch, Y. Pan, and W. Peelaers, Intersecting Surface Defects and Two-Dimensional CFT, arXiv:1610.0350.

[35] E. Witten, Fivebranes and knots, Quantum Topol. 3 (2012) 1-137, [1101.3216].

[36] N. A. Nekrasov and S. L. Shatashvili, Quantization of Integrable Systems and Four Dimensional Gauge Theories, in Proceedings, 16th International Congress on Mathematical Physics (ICMP09): Prague, Czech Republic, August 3-8, 2009, pp. 265-289, 2009. arXiv:0908.4052,

[37] G. Vartanov and J. Teschner, Supersymmetric gauge theories, quantization of moduli spaces of flat connections, and conformal field theory, Adv. Theor. Math. Phys. 19 (2015) 1-135, [arXiv:1302.3778].

[38] T. Fujimori, T. Kimura, M. Nitta, and K. Ohashi, $2 d$ partition function in $\Omega$-background and vortex/instanton correspondence, JHEP 12 (2015) 110, [arXiv:1509.0863].

[39] D. Honda and T. Okuda, Exact results for boundaries and domain walls in $2 d$ supersymmetric theories, JHEP 09 (2015) 140, [arXiv: 1308.2217].

[40] K. Hori and M. Romo, Exact Results In Two-Dimensional $(2,2)$ Supersymmetric Gauge Theories With Boundary, arXiv:1308.2438,

[41] K. Hori, A. Iqbal, and C. Vafa, D-branes and mirror symmetry, hep-th/0005247,

[42] S. Cecotti and C. Vafa, Topological antitopological fusion, Nucl. Phys. B367 (1991) 359-461.

[43] D. Gaiotto, Surface Operators in N = 2 4d Gauge Theories, JHEP 11 (2012) 090, [arXiv:0911.1316].

[44] O. Dumitrescu, L. Fredrickson, G. Kydonakis, R. Mazzeo, M. Mulase, and A. Neitzke, Opers versus nonabelian Hodge, ArXiv e-prints (July, 2016) [arXiv: 1607.0217].

[45] N. J. Hitchin, The self-duality equations on a Riemann surface, Proc. London Math. Soc. (3) 55 (1987), no. 1 59-126. 
[46] D. Gaiotto, Opers and TBA, arXiv:1403.6137.

[47] E. Witten, More On Gauge Theory And Geometric Langlands, arXiv:1506.0429.

[48] D. Gaiotto, S-duality of boundary conditions and the Geometric Langlands program, arXiv:1609.0903.

[49] D. Gaiotto, Twisted compactifications of $3 d N=4$ theories and conformal blocks, arXiv:1611.0152.

[50] C. Simpson, Higgs bundles and local systems, Publ. Math. IHES 75 (1992) 5-95. 